\title{
A Non-parametric Approach for Uncertainty Quantification in Elastodynamics
}

\author{
Sondipon Adhikari* \\ University of Bristol, Bristol, United Kingdom
}

\begin{abstract}
Matrix variate distributions are are used to quantify uncertainty in the mass, stiffness and damping matrices. The proposed approach is based on the so called Wishart random matrices. The probability density function of the system matrices are derived using the maximum entropy method. It is assumed that the mean of the system matrices are known. A new optimal Wishart distribution is proposed to model the random system matrices. The optimal Wishart distribution is such that the mean of the matrix and its inverse produce minimum deviations from their deterministic values. The method proposed here gives a simple nonparametric approach for uncertainty quantification and propagation for complex aerospace structural systems. The new method is illustrated using two examples.
\end{abstract}

\section{Nomenclature}

$\begin{array}{ll}\mathbf{D}(\omega) & \text { dynamic stiffness matrix } \\ \mathbf{F} & \text { symbol for the inverse of a system matrix, } \mathbf{F} \equiv\left\{\mathbf{M}^{-1}, \mathbf{C}^{-1}, \mathbf{K}^{-1}\right\} \\ \mathbf{f}(t) & \text { forcing vector } \\ \mathbf{G} & \text { symbol for a system matrix, } \mathbf{G} \equiv\{\mathbf{M}, \mathbf{C}, \mathbf{K}\} \\ \mathbf{H}(\omega) & \text { frequency response function }(\mathrm{FRF}) \text { matrix } \\ \mathbf{I}_{n} & \text { identity matrix of dimension } n \\ \mathbf{M}, \mathbf{C} \text { and } \mathbf{K} & \text { mass, damping and stiffness matrices respectively } \\ \mathbf{O}_{n, m} & \text { null matrix of dimension } n \times m \\ \mathbf{q}(t) & \text { response vector } \\ \Gamma_{n}(a) & \text { multivariate gamma function } \\ \nu & \text { order of the inverse-moment constraint } \\ \omega & \text { excitation frequency } \\ m, \mathbf{\Psi} & \text { scalar and matrix parameters of the inverted Wishart distribution } \\ n & \text { number of degrees of freedom } \\ p, \boldsymbol{\Sigma} & \text { scalar and matrix parameters of the Wishart distribution }\end{array}$

Conventions

$\begin{array}{ll}(\bullet)^{T} & \text { matrix transposition } \\ \mathbb{C} & \text { space of complex numbers } \\ \mathbb{R} & \text { space of real numbers } \\ \mathbb{R}_{n}^{+} & \text {space } n \times n \text { real positive definite matrices } \\ \mathbb{R}_{n, m} & \text { space } n \times m \text { real matrices }\end{array}$

${ }^{*}$ Lecturer, Department of Aerospace Engineering, University of Bristol, Queens Building, University Walk, Bristol BS8 1TR, UK, AIAA Member.

1 of 21 


$\begin{array}{ll}|\bullet| & \text { determinant of a matrix } \\ \text { etr }\{\bullet\} & \text { exp }\{\operatorname{Trace}(\bullet)\} \\ \mathrm{E}[\bullet] & \text { expectation operator } \\ \|\bullet\|_{\mathrm{F}} & \text { Frobenius norm of a matrix, }\|\bullet\|_{\mathrm{F}}=\left(\operatorname{Trace}\left((\bullet)(\bullet)^{T}\right)\right)^{1 / 2} \\ \otimes & \text { Kronecker product }\left(\sec ^{1}\right) \\ \sim & \text { distributed as } \\ \text { Trace }(\bullet) & \text { sum of the diagonal elements of a matrix } \\ p_{(\bullet)}(\mathbf{X}) & \text { probability density function of }(\bullet) \text { in (matrix) variable } \mathbf{X} \\ \text { pdf } & \text { probably density function }\end{array}$

\section{Introduction}

Uncertainties are unavoidable in the description of real-life engineering systems. The quantification of uncertainties plays a crucial role in establishing the credibility of a numerical model. Uncertainties can be broadly divided into two categories. The first type is due to the inherent variability in the system parameters, for example, different cars manufactured from a single production line are not exactly the same. This type of uncertainty is often referred to as aleatoric uncertainty. If enough samples are present, it is possible to characterize the variability using well established statistical methods and consequently the probably density functions (pdf) of the parameters can be obtained. The second type of uncertainty is due to the lack of knowledge regarding a system, often referred to as epistemic uncertainty. This kind of uncertainty generally arise in the modelling of complex systems, for example, in the modeling of cabin noise in helicopters. Due to its very nature, it is comparatively difficult to quantify or model this type of uncertainties.

Broadly speaking, there are two approaches to quantify uncertainties in a model. The first is the parametric approach and the second is the non-parametric approach. In the parametric approach the uncertainties associates with the system parameters, such as Young's modulus, mass density, Poisson's ratio, damping coefficient and geometric parameters are quantified using statistical methods and propagated, for example, using the stochastic finite element method. This type of approach is suitable to quantify aleatoric uncertainties. Epistemic uncertainty on the other hand do not explicitly depend on the systems parameters. For example, there can be unquantified errors associated with the equation of motion (linear on non-linear), in the damping model (viscous or non-viscous), in the model of structural joints, and also in the numerical methods (e.g, discretisation of displacement fields, truncation and roundoff errors, tolerances in the optimization and iterative algorithms, step-sizes in the time-integration methods). It is evident that the parametric approach is not suitable to quantify this type of uncertainties and a non-parametric approach is needed for this purpose. The aim of this paper is to develop a general non-parametric uncertainty quantification tool for structural dynamic systems. The proposed approach is based on the random matrix theory and the maximum entropy method.

Uncertainties associated with a variable can be characterized using the probabilistic approach or possibilistic approaches based on interval algebra, convex sets, Fuzzy sets or generalized DempsterSchafer theory. In this paper probabilistic approach has been adopted. The equation of motion of a damped $n$-degree-of-freedom linear dynamic system can be expressed as

$$
\mathbf{M} \ddot{\mathbf{q}}(t)+\mathbf{C} \dot{\mathbf{q}}(t)+\mathbf{K q}(t)=\mathbf{f}(t)
$$

where $\mathbf{M}, \mathbf{C}$ and $\mathbf{K}$ are the mass, damping and stiffness matrices respectively. The importance of considering parametric and/or non-parametric uncertainty also depends on vibration frequency of excitation. For example, in the high frequency vibration the wave lengths of the vibration modes can become very small. As a result the vibration response can be very sensitive to the small details of the system. In such situations a non-parametric uncertainty model may be adequate. Overall, 
three different approaches are currently available to analyze stochastic structural dynamic systems across the frequency range:

- Low frequency vibration problems: Stochastic Finite Element Method ${ }^{2-4}$ (SFEM) - considers parametric uncertainties in details;

- High frequency vibration problems: Statistical Energy Analysis ${ }^{5,6}$ (SEA) - do not consider parametric uncertainties in details;

- Mid-frequency vibration problems: Hybrid method, ${ }^{7}$ substructure approach ${ }^{8}$.

The aim of this paper is to propose a method which will work across the frequency range. The probability density functions of the random matrices $\mathbf{M}, \mathbf{C}$ and $\mathbf{K}$ are derived to to completely quantify the uncertainties associated with system (1). In the next section we briefly outline some aspects of random matrix theory required for further developments.

\section{Background of the Random Matrix Theory}

Random matrices were introduced by $\mathrm{Wishart}^{9}$ in the late $1920 \mathrm{~s}$ in the context of multivariate statistics. However, random matrix theory (RMT) was not used in other branches until 1950s when Wigner ${ }^{10}$ published his works on the eigenvalues of random matrices arising in high-energy physics. Using an asymptotic theory for large dimensional matrices, Wigner was able to bypass the Schrödinger equation and explain the statistics of measured atomic energy levels in terms of the limiting spectrum of these random matrices. Since then, research on random matrices has continued to attract interest in multivariate statistics, physics, number theory and more recently in mechanical and electrical engineering. We refer the readers to the books by Mezzadri and Snaith, ${ }^{11}$ Tulino and Verdú, ${ }^{12}$ Muirhead ${ }^{13}$ and Mehta $^{14}$ for history and applications Random Matrix Theory.

The probability density function of a random matrix can be defined in a manner similar to that of a random variable. If $\mathbf{A}$ is an $n \times m$ real random matrix, the matrix variate probability density function of $\mathbf{A} \in \mathbb{R}_{n, m}$, denoted as $p_{\mathbf{A}}(\mathbf{A})$, is a mapping from the space of $n \times m$ real matrices to the real line, i.e., $p_{\mathbf{A}}(\mathbf{A}): \mathbb{R}_{n, m} \rightarrow \mathbb{R}$. Here we define four types of random matrices which are relevant to this study.

Definition 1. Gaussian random matrix: The random matrix $\mathbf{X} \in \mathbb{R}_{n, p}$ is said to have a matrix variate Gaussian distribution with mean matrix $\mathbf{M} \in \mathbb{R}_{n, p}$ and covariance matrix $\boldsymbol{\Sigma} \otimes \mathbf{\Psi}$, where $\boldsymbol{\Sigma} \in \mathbb{R}_{n}^{+}$and $\boldsymbol{\Psi} \in \mathbb{R}_{p}^{+}$provided the pdf of $\mathbf{X}$ is given by

$$
p_{\mathbf{X}}(\mathbf{X})=(2 \pi)^{-n p / 2}|\boldsymbol{\Sigma}|^{-p / 2}|\boldsymbol{\Psi}|^{-n / 2} \operatorname{etr}\left\{-\frac{1}{2} \boldsymbol{\Sigma}^{-1}(\mathbf{X}-\mathbf{M}) \boldsymbol{\Psi}^{-1}(\mathbf{X}-\mathbf{M})^{T}\right\}
$$

This distribution is usually denoted as $\mathbf{X} \sim N_{n, p}(\mathbf{M}, \boldsymbol{\Sigma} \otimes \mathbf{\Psi})$.

Definition 2. Gaussian orthogonal ensembles (GOE): A random matrix $\mathbf{H} \in \mathbb{R}_{n, n}$ belongs to the Gaussian orthogonal ensemble (GOE) provided its pdf is given by

$$
p_{\mathbf{H}}(\mathbf{H})=\exp \left(-\theta_{2} \operatorname{Trace}\left(\mathbf{H}^{2}\right)+\theta_{1} \operatorname{Trace}(\mathbf{H})+\theta_{0}\right)
$$

where $\theta_{2}$ is real and positive and $\theta_{1}$ and $\theta_{0}$ are real.

Definition 3. Wishart matrix: An $n \times n$ random symmetric positive definite matrix $\mathbf{S}$ is said to have a Wishart distribution with parameters $p \geq n$ and $\boldsymbol{\Sigma} \in \mathbb{R}_{n}^{+}$, if its pdf is given by

$$
p_{\mathbf{S}}(\mathbf{S})=\left\{2^{\frac{1}{2} n p} \Gamma_{n}\left(\frac{1}{2} p\right)|\boldsymbol{\Sigma}|^{\frac{1}{2} p}\right\}^{-1}|\mathbf{S}|^{\frac{1}{2}(p-n-1)} \operatorname{etr}\left\{-\frac{1}{2} \boldsymbol{\Sigma}^{-1} \mathbf{S}\right\}
$$

This distribution is usually denoted as $\mathbf{S} \sim W_{n}(p, \boldsymbol{\Sigma})$. 
Definition 4. Matrix variate gamma distribution: An $n \times n$ random symmetric positive definite matrix $\mathbf{W}$ is said to have a matrix variate gamma distribution with parameters $a$ and $\Psi \in \mathbb{R}_{n}^{+}$, if its pdf is given by

$$
p_{\mathbf{W}}(\mathbf{W})=\left\{\Gamma_{n}(a)|\Psi|^{-a}\right\}^{-1}|\mathbf{W}|^{a-\frac{1}{2}(n+1)} \operatorname{etr}\{-\mathbf{\Psi} \mathbf{W}\} ; \quad \Re(a)>\frac{1}{2}(n-1)
$$

This distribution is usually denoted as $\mathbf{W} \sim G_{n}(a, \mathbf{\Psi})$. The matrix variate gamma distribution was used by Soize ${ }^{15-17}$ for the random system matrices of linear dynamical systems.

Definition 5. Inverted Wishart distribution: An $n \times n$ random symmetric positive definite matrix $\mathbf{V}$ is said to have a inverted Wishart distribution with parameters $m$ and $\boldsymbol{\Psi} \in \mathbb{R}_{n}^{+}$, if its pdf is given by

$$
p_{\mathbf{V}}(\mathbf{V})=\frac{2^{-\frac{1}{2}(m-n-1) n \mid}|\boldsymbol{\Psi}|^{\frac{1}{2}(m-n-1)}}{\Gamma_{n}\left(\frac{1}{2}(m-n-1)\right)|\mathbf{V}|^{m / 2}} \operatorname{etr}\left\{-\mathbf{V}^{-1} \boldsymbol{\Psi}\right\} ; \quad m>2 n, \boldsymbol{\Psi}>0 .
$$

This distribution is usually denoted as $\mathbf{V} \sim I W_{n}(m, \mathbf{\Psi})$.

In Eqs. (4)-(6), the function $\Gamma_{n}(a)$ is the multivariate gamma function, which can be expressed as a product of univariate gamma functions as

$$
\Gamma_{n}(a)=\pi^{\frac{1}{4} n(n-1)} \prod_{k=1}^{n} \Gamma\left[a-\frac{1}{2}(k-1)\right] ; \quad \text { for } \quad \Re(a)>\frac{1}{2}(n-1)
$$

For more details on the matrix variate distributions we refer to the books by Tulino and Verdú, ${ }^{12}$ Gupta and Nagar, ${ }^{18}$ Muirhead $^{13}$ and references therein.

\section{Matrix Variate Distribution for Structural Mechanics}

In this section an information theoretic approach is taken to obtain the matrix variate distributions of the random system matrices $\mathbf{M}, \mathbf{C}$ and $\mathbf{K}$. First we look at the information available to us and then consider the constraints the matrix variate distributions must satisfy in order to be physically realistic. Once these steps are completed, the matrix variate distributions will be obtained using the maximum entropy method. In a series of papers Soize ${ }^{15-17}$ used this approach to obtain the probability density function of the system matrices.

Suppose that the mean values of $\mathbf{M}, \mathbf{C}$ and $\mathbf{K}$ are given by $\overline{\mathbf{M}}, \overline{\mathbf{C}}$ and $\overline{\mathbf{K}}$ respectively. This information is likely to be available, for example, using the deterministic finite element method. However, there are uncertainties associated with our modelling so that $\mathbf{M}, \mathbf{C}$ and $\mathbf{K}$ are actually random matrices. The distribution of these random matrices should be such that they are

(a) symmetric

(b) positive-definite, and

(c) the moments of the inverse of the dynamic stiffness matrix

$$
\mathbf{D}(\omega)=-\omega^{2} \mathbf{M}+i \omega \mathbf{C}+\mathbf{K}
$$

should exist $\forall \omega$. That is, if $\mathbf{H}(\omega)$ is the frequency response function (FRF) matrix

$$
\mathbf{H}(\omega)=\mathbf{D}^{-1}(\omega)=\left[-\omega^{2} \mathbf{M}+i \omega \mathbf{C}+\mathbf{K}\right]^{-1}
$$

then the following condition must be satisfied:

$$
\mathrm{E}\left[\|\mathbf{H}(\omega)\|_{\mathrm{F}}^{\nu}\right]<\infty, \quad \forall \omega
$$


Here $\nu$ is the order of the inverse-moment constraint. For example if $\mathbf{H}(\omega)$ is considered to be a second-order (matrix variate) random process then $\nu=2$ should be used. This constraint clearly arising from the fact that the moments and the pdf of the response vector must exist for all frequency of excitation. Because the matrices $\mathbf{M}, \mathbf{C}$ and $\mathbf{K}$ have similar probabilistic characteristics, for notational convenience we will use the notation $\mathbf{G}$ which stands for any one the system matrices. Suppose the matrix variate density function of $\mathbf{G} \in \mathbb{R}_{n}^{+}$is given by $p_{\mathbf{G}}(\mathbf{G}): \mathbb{R}_{n}^{+} \rightarrow \mathbb{R}$. We have the following information and constrains to obtain $p_{\mathbf{G}}(\mathbf{G})$ :

$$
\begin{gathered}
\int_{\mathbf{G}>0} p_{\mathbf{G}}(\mathbf{G}) d \mathbf{G}=1 \quad \text { (normalization) } \\
\text { and } \int_{\mathbf{G}>0} \mathbf{G} p_{\mathbf{G}}(\mathbf{G}) d \mathbf{G}=\overline{\mathbf{G}} \quad \text { (the mean matrix) }
\end{gathered}
$$

The mean matrix $\overline{\mathbf{G}}$ is symmetric and positive definite and the integrals appearing in the above two equations are $n(n+1) / 2$ dimensional. The maximum entropy method ${ }^{19}$ can be used to obtain the probability density function of the random system matrices. Using this approach, Soize ${ }^{15-17}$ obtained the matrix variate gamma distribution given by Eq. (5) for $\mathbf{G}$ with parameters $a=$ $\lambda+\frac{1}{2}(n-1)$ and $\boldsymbol{\Psi}=\left\{\lambda+\frac{1}{2}(n-1)\right\} \overline{\mathbf{G}}^{-1}$ where $\lambda \in \mathbb{R}$ is an undetermined coefficient. The main difference between the matrix variate gamma distribution and the Wishart distribution is that historically only integer values were considered for the shape parameter $p$ in the Wishart matrices. However, from an analytical point of view the gamma and the Wishart distributions are identical and the modern random matrix theory normally does not make any distinctions between them. Since the Wishart random matrices are used widely in different subjects, ${ }^{12,13}$ in this paper we present our results in terms of the Wishart matrices. In the next subsections, the matrix variate distributions are derived considering two separate cases.

\section{A. Distribution Without the Inverse-Moment Constraint}

The inverse-moment constraint of the dynamic stiffness matrix mentioned before (condition (c)) is difficult to implement analytically. Therefore, in this section we derive the pdf of the system matrices without this constraint. This distribution is 'maximally uncertain' because minimum number of constraints are used to derive this distribution. Once the distribution is obtained, the consequence of ignoring the inverse-moment constraint will be discussed. In order to extend the maximum entropy method to random matrices, first note that the entropy associated with the matrix variate probability density function $p_{\mathbf{G}}(\mathbf{G})$ can be expressed as

$$
\mathcal{S}\left(p_{\mathbf{G}}\right)=-\int_{\mathbf{G}>0} p_{\mathbf{G}}(\mathbf{G}) \ln \left\{p_{\mathbf{G}}(\mathbf{G})\right\} d \mathbf{G}
$$

Using this, together with the constrains in Eqs. (11) and (12) we construct the Lagrangian

$$
\begin{aligned}
\mathcal{L}\left(p_{\mathbf{G}}\right)=-\int_{\mathbf{G}>0} p_{\mathbf{G}}(\mathbf{G}) \ln \left\{p_{\mathbf{G}}(\mathbf{G})\right\} d \mathbf{G}+\left(\lambda_{0}-1\right) & \left(\int_{\mathbf{G}_{>0}} p_{\mathbf{G}}(\mathbf{G}) d \mathbf{G}-1\right) \\
& +\operatorname{Trace}\left(\boldsymbol{\Lambda}_{1}\left[\int_{\mathbf{G}>0} \mathbf{G} p_{\mathbf{G}}(\mathbf{G}) d \mathbf{G}-\overline{\mathbf{G}}\right]\right)
\end{aligned}
$$

Here $\lambda_{0} \in \mathbb{R}$ and $\boldsymbol{\Lambda}_{1} \in \mathbb{R}_{n, n}$ are the unknown Lagrange multiplies which need to be determined. Using the variational calculus it can be shown that the optimal condition is given by

$$
\begin{array}{ll} 
& \frac{\partial \mathcal{L}\left(p_{\mathbf{G}}\right)}{\partial p_{\mathbf{G}}}=0 \quad \text { or } \quad-\left(1+\ln \left\{p_{\mathbf{G}}(\mathbf{G})\right\}\right)+\left(\lambda_{0}-1\right)+\operatorname{Trace}\left(\boldsymbol{\Lambda}_{1} \mathbf{G}\right)=0 \\
\text { or } \quad-\ln \left\{p_{\mathbf{G}}(\mathbf{G})\right\}=\lambda_{0}+\operatorname{Trace}\left(\boldsymbol{\Lambda}_{1} \mathbf{G}\right) \\
\text { or } \quad p_{\mathbf{G}}(\mathbf{G})=\exp \left\{-\lambda_{0}\right\} \operatorname{etr}\left\{-\boldsymbol{\Lambda}_{1} \mathbf{G}\right\}
\end{array}
$$


Using the matrix calculus, ${ }^{20,21}$ the Lagrange multipliers $\lambda_{0}$ and $\boldsymbol{\Lambda}_{1}$ can be obtained exactly from equations (11), (12) and (15) to obtain $p_{\mathbf{G}}(\mathbf{G})$. After some algebra it can be shown that

$$
p_{\mathbf{G}}(\mathbf{G})=r^{-n r}\left\{\Gamma_{n}(r)\right\}^{-1}|\overline{\mathbf{G}}|^{-r} \operatorname{etr}\left\{-r \overline{\mathbf{G}}^{-1} \mathbf{G}\right\}, \quad \text { where } \quad r=\frac{1}{2}(n+1)
$$

Comparing Eq. (16) with the Wishart distribution in Eq. (4) it can be observed that $\mathbf{G}$ has the Wishart distribution with parameters $p=n+1$ and $\boldsymbol{\Sigma}=\overline{\mathbf{G}} /(n+1)$. Therefore, we have the following fundamental result regarding the non-parametric uncertainty modeling of structural dynamic systems:

Theorem 1. If only the mean of a system matrix $\mathbf{G} \equiv\{\mathbf{M}, \mathbf{C}, \mathbf{K}\}$ is available, say $\overline{\mathbf{G}}$, then the maximum-entropy pdf of $\mathbf{G}$ follows the Wishart distribution with parameters $(n+1)$ and $\overline{\mathbf{G}} /(n+1)$, that is $\mathbf{G} \sim W_{n}(n+1, \overline{\mathbf{G}} /(n+1))$.

\section{B. Distribution With the Inverse-Moment Constraint}

In the previous section we have ignored the constraint that the inverse-moments of the dynamic stiffness matrix should exist for all frequencies. The exact application of this constraint requires the derivation of the joint probability density function of the random matrices $\mathbf{M}, \mathbf{C}$ and $\mathbf{K}$, which is analytically untractable within the scope of the present state of developments in the random matrix theory. Therefore, we consider a simpler problem where it is required that inverse moments of each of the systems matrices $\mathbf{M}, \mathbf{C}$ and $\mathbf{K}$ must exist. ${ }^{15-17}$ Provided the system is damped, this condition will always guarantee the existence of the moments of the frequency response function matrix. This condition is only a sufficient condition and not a necessary condition. As a result, the distributions arising from this approach will be more constrained than that is necessary.

Suppose the inverse moments (say up to order $\nu$ ) of a system matrix exist. This implies that $\mathrm{E}\left[\left\|\mathbf{G}^{-1}\right\|_{\mathrm{F}}{ }^{\nu}\right]$ should be finite. Because $\mathbf{G}$ is a symmetric positive-definite matrix, $\mathbf{G}$ can be expressed in terms of its eigenvalues and eigenvectors as $\mathbf{G}=\boldsymbol{\Phi} \boldsymbol{\lambda} \boldsymbol{\Phi}^{T}$. Here $\boldsymbol{\Phi} \in \mathbb{R}_{n, n}$ is an orthonormal matrix containing the eigenvectors of $\mathbf{G}$, that ie, $\boldsymbol{\Phi}^{T} \boldsymbol{\Phi}=\mathbf{I}_{n}$ and $\boldsymbol{\lambda}$ is a real diagonal matrix containing the eigenvalues of $\mathbf{G}$. Note that for any $\alpha \in \mathbb{R}, \mathbf{G}^{\alpha}=\boldsymbol{\Phi} \boldsymbol{\lambda}^{\alpha} \boldsymbol{\Phi}^{T}$. Recalling that the Frobenius norm of the matrix $\mathbf{G}$ is given by $\|\mathbf{G}\|_{\mathrm{F}}=\left(\operatorname{Trace}\left(\mathbf{G G}^{T}\right)\right)^{1 / 2}$ and for any three compatible matrices Trace $\left(\mathbf{A}_{1} \mathbf{A}_{2} \mathbf{A}_{3}\right)=\operatorname{Trace}\left(\mathbf{A}_{3} \mathbf{A}_{1} \mathbf{A}_{2}\right)=\operatorname{Trace}\left(\mathbf{A}_{2} \mathbf{A}_{3} \mathbf{A}_{1}\right)$, we have

$$
\begin{aligned}
\left\|\mathbf{G}^{-1}\right\|_{\mathrm{F}}{ }^{\nu} & =\operatorname{Trace}\left(\sqrt{\boldsymbol{\Phi} \boldsymbol{\lambda}^{-\nu} \boldsymbol{\Phi}^{T} \boldsymbol{\Phi} \boldsymbol{\lambda}^{-\nu} \boldsymbol{\Phi}^{T}}\right)=\operatorname{Trace}\left(\boldsymbol{\Phi} \boldsymbol{\lambda}^{-\nu} \boldsymbol{\Phi}^{T}\right)=\operatorname{Trace}\left(\boldsymbol{\Phi}^{T} \boldsymbol{\Phi} \boldsymbol{\lambda}^{-\nu}\right) \\
& =\operatorname{Trace}\left(\boldsymbol{\lambda}^{-\nu}\right)=\lambda_{1}^{-\nu}+\lambda_{2}^{-\nu}+\cdots \lambda_{n}^{-\nu}
\end{aligned}
$$

Because all the eigenvalues are positive, the condition $\mathrm{E}\left[\left\|\mathrm{G}^{-1}\right\|_{\mathrm{F}}{ }^{\nu}\right]<\infty$ will also be satisfied when

$$
\begin{aligned}
& \mathrm{E}\left[\ln \lambda_{1}^{-\nu}+\ln \lambda_{2}^{-\nu}+\cdots \ln \lambda_{n}^{-\nu}\right]<\infty \\
\text { or } \quad & \mathrm{E}\left[-\nu \ln \left(\lambda_{1} \lambda_{2} \cdots \lambda_{n}\right)\right]<\infty \\
\text { or } \quad & \mathrm{E}\left[\ln |\mathbf{G}|^{-\nu}\right]<\infty
\end{aligned}
$$

Equation (18) is the new constraint which should be satisfied. Therefore, the Lagrangian becomes

$$
\begin{aligned}
& \mathcal{L}\left(p_{\mathbf{G}}\right)=-\int_{\mathbf{G}>0} p_{\mathbf{G}}(\mathbf{G}) \ln \left\{p_{\mathbf{G}}(\mathbf{G})\right\} d \mathbf{G}+\left(\lambda_{0}-1\right)\left(\int_{\mathbf{G}_{>0}} p_{\mathbf{G}}(\mathbf{G}) d \mathbf{G}-1\right)- \\
& \nu \int_{\mathbf{G}>0} \ln |\mathbf{G}| p_{\mathbf{G}} d \mathbf{G}+\operatorname{Trace}\left(\boldsymbol{\Lambda}_{1}\left[\int_{\mathbf{G}_{>0}} \mathbf{G} p_{\mathbf{G}}(\mathbf{G}) d \mathbf{G}-\overline{\mathbf{G}}\right]\right)
\end{aligned}
$$

6 of 21

American Institute of Aeronautics and Astronautics 
Although $\nu$ behaves like a Lagrange multiplier, it cannot be obtained uniquely because the constrain in Eq. (18) in not finite. As a result we treat $\nu$ as a parameter in the optimization procedure. Again, using the calculus of variation we have

$$
\begin{aligned}
& \quad \frac{\partial \mathcal{L}\left(p_{\mathbf{G}}\right)}{\partial p_{\mathbf{G}}}=0 \\
& \text { or }-\ln \left\{p_{\mathbf{G}}(\mathbf{G})\right\}=\lambda_{0}+\operatorname{Trace}\left(\boldsymbol{\Lambda}_{1} \mathbf{G}\right)-\ln |\mathbf{G}|^{\nu} \\
& \text { or } p_{\mathbf{G}}(\mathbf{G})=\exp \left\{-\lambda_{0}\right\}|\mathbf{G}|^{\nu} \operatorname{etr}\left\{-\boldsymbol{\Lambda}_{1} \mathbf{G}\right\}
\end{aligned}
$$

Using the expression of the matrix variate Laplace transform ${ }^{18}$

$$
\int_{\mathbf{T}>0} \operatorname{etr}\{-\mathbf{S T}\}|\mathbf{T}|^{a-(n+1) / 2} d \mathbf{T}=\Gamma_{n}(a)|\mathbf{S}|^{-a}, \quad \mathbf{T} \in \mathbb{R}_{n, n}, \mathbf{S} \in \mathbb{C}_{n, n}, a>(n+1) / 2
$$

and substituting $p_{\mathbf{G}}(\mathbf{G})$ from Eq. (20) into the constraint equations (11) and (12), the Lagrange multipliers $\lambda_{0}$ and $\boldsymbol{\Lambda}_{1}$ can be obtained exactly. After some algebra it can be again shown that

$$
p_{\mathbf{G}}(\mathbf{G})=r^{-n r}\left\{\Gamma_{n}(r)\right\}^{-1}|\overline{\mathbf{G}}|^{-r}|\mathbf{G}|^{\nu} \operatorname{etr}\left\{-r \overline{\mathbf{G}}^{-1} \mathbf{G}\right\}, \quad \text { where } \quad r=\nu+\frac{1}{2}(n+1)
$$

Comparing Eq. (22) with the Wishart distribution in Eq. (4) it can be observed that $\mathbf{G}$ has the Wishart distribution with parameters $p=2 \nu+n+1$ and $\boldsymbol{\Sigma}=\overline{\mathbf{G}} /(2 \nu+n+1)$. Therefore, we have the following basic result regarding the non-parametric uncertainty modeling of structural dynamic systems:

Theorem 2. If $\nu$-th order inverse-moment of a system matrix $\mathbf{G} \equiv\{\mathbf{M}, \mathbf{C}, \mathbf{K}\}$ exists and only the mean of $\mathbf{G}$ is available, say $\overline{\mathbf{G}}$, then the maximum-entropy pdf of $\mathbf{G}$ follows the Wishart distribution with parameters $p=(2 \nu+n+1)$ and $\mathbf{\Sigma}=\overline{\mathbf{G}} /(2 \nu+n+1)$, that is $\mathbf{G} \sim W_{n}(2 \nu+n+1, \overline{\mathbf{G}} /(2 \nu+n+1))$.

Observe that $\nu=0$ gives us the 'maximal uncertain distribution' derived in the previous subsection.

\section{Statistical Properties of the System Matrices}

From the discussion in the previous section it is clear that each system matrix follows a Wishart distribution. The discovery of the Wishart distribution in this context turns out to be very useful because it has been studied extensively in the multivariate statistics literature. ${ }^{13,18,22}$ Here we outline some interesting properties of the distribution. For convenience we present the results in terms of the general Wishart matrix $\mathbf{G} \sim W_{n}(p, \boldsymbol{\Sigma})$, where, $\boldsymbol{\Sigma}=\overline{\mathbf{G}} / p$ and $p=2 \nu+n+1$ or $p=n+1$ depending on whether the inverse-moment constraint is considered or not.

Perhaps the most useful property of a Wishart matrix is the fact that it can be decomposed in terms of Gaussian random matrices:

Theorem 3. If $\mathbf{X}$ is a Gaussian random matrix such that $\mathbf{X} \sim N_{n, p}\left(\mathbf{O}_{n, p}, \boldsymbol{\Sigma} \otimes \mathbf{I}_{p}\right)$, then $\mathbf{G}=\mathbf{X X}^{T}$ has the Wishart distribution $\mathbf{G} \sim W_{n}(p, \boldsymbol{\Sigma})$.

The proof is available, for example, in reference. ${ }^{18}$ This result is particularly important because it gives an easy simulation algorithm for the system matrices. From this representation, it is clear that $\mathbf{G}$ is symmetric with probability one. Using Theorem 3.2.1 of Gupta and Nagar ${ }^{18}$ we can also say that $\mathbf{G}$ is positive definite with probability one. Therefore, two out of the three requirements outlined in the previous subsections are always satisfied by a Wishart matrix.

7 of 21

American Institute of Aeronautics and Astronautics 
The probability density function of $\mathbf{G}$ has been derived exactly in closed-form. Now we use them to obtain the moments of the random matrix $\mathbf{G}$. The characteristic function of $\mathbf{G}$, that is the joint characteristic function of $G_{11}, G_{12}, G_{n n}$, can be obtained as

$$
\Phi_{\mathbf{G}}(\mathbf{Z})=\mathrm{E}[\operatorname{etr}\{\mathrm{i} \mathbf{Z G}\}]=\left|\mathbf{I}_{n}-\mathrm{i} \mathbf{Z} \mathbf{\Sigma}\right|^{-p / 2}
$$

where $\mathbf{Z} \in \mathbb{R}_{n, n}$ is a symmetric matrix. The first moment (mean), second-moment, the elements of the covariance tensor and the variance of $\mathbf{G}$ can be obtained ${ }^{18}$ as

$$
\begin{aligned}
& \mathrm{E}[\mathbf{G}]=p \boldsymbol{\Sigma}=\overline{\mathbf{G}} \\
& \mathrm{E}\left[\mathbf{G}^{2}\right]=p \boldsymbol{\Sigma}^{2}+p \operatorname{Trace}(\boldsymbol{\Sigma}) \boldsymbol{\Sigma}+p^{2} \boldsymbol{\Sigma}^{2}=\frac{1}{2 \nu+n+1}\left[(2 \nu+n+2) \overline{\mathbf{G}}^{2}+\overline{\mathbf{G}} \text { Trace }(\overline{\mathbf{G}})\right] \\
& \operatorname{cov}\left(G_{i j}, G_{k l}\right)=p\left(\Sigma_{i k} \Sigma_{j l}+\Sigma_{i l} \Sigma_{j k}\right)=\frac{1}{2 \nu+n+1}\left(\bar{G}_{i k} \bar{G}_{j l}+\bar{G}_{i l} \bar{G}_{j k}\right) \\
& \mathrm{E}\left[\{\mathbf{G}-\mathrm{E}[\mathbf{G}]\}^{2}\right]=\mathrm{E}\left[\mathbf{G}^{2}\right]-\overline{\mathbf{G}}^{2}=\frac{1}{2 \nu+n+1}\left[\overline{\mathbf{G}}^{2}+\overline{\mathbf{G}} \text { Trace }(\overline{\mathbf{G}})\right]
\end{aligned}
$$

It is useful to define the normalized standard deviation of $\mathbf{G}$ (introduced by Soize ${ }^{15-17}$ as the dispersion parameter) as

$$
\delta_{G}^{2}=\frac{\mathrm{E}\left[\|\mathbf{G}-\mathrm{E}[\mathbf{G}]\|_{\mathrm{F}}^{2}\right]}{\|\mathrm{E}[\mathbf{G}]\|_{\mathrm{F}}^{2}}
$$

Because both E $[\bullet]$ and Trace $(\bullet)$ are linear operators their order can be interchanged. Now using Eqs. (24) and (25) we have

$$
\begin{aligned}
\mathrm{E}\left[\|\mathbf{G}-\mathrm{E}[\mathbf{G}]\|_{\mathrm{F}}^{2}\right] & =\mathrm{E}\left[\operatorname{Trace}\left([\mathbf{G}-\mathrm{E}[\mathbf{G}]][\mathbf{G}-\mathrm{E}[\mathbf{G}]]^{T}\right)\right] \\
& =\operatorname{Trace}\left(\mathrm{E}\left[\left[\mathbf{G}^{2}-\mathbf{G E}[\mathbf{G}]-\mathrm{E}[\mathbf{G}] \mathbf{G}-\mathrm{E}[\mathbf{G}]^{2}\right]\right]\right)=\operatorname{Trace}\left(\mathrm{E}\left[\mathbf{G}^{2}\right]-\mathrm{E}[\mathbf{G}]^{2}\right) \\
& =\operatorname{Trace}\left(p \boldsymbol{\Sigma}^{2}+p \operatorname{Trace}(\boldsymbol{\Sigma}) \boldsymbol{\Sigma}+p^{2} \boldsymbol{\Sigma}^{2}-(p \boldsymbol{\Sigma})^{2}\right) \\
& =p \operatorname{Trace}\left(\boldsymbol{\Sigma}^{2}\right)+p\{\operatorname{Trace}(\boldsymbol{\Sigma})\}^{2}
\end{aligned}
$$

Therefore

$$
\begin{aligned}
\delta_{G}^{2} & =\frac{p \operatorname{Trace}\left(\boldsymbol{\Sigma}^{2}\right)+p\{\operatorname{Trace}(\boldsymbol{\Sigma})\}^{2}}{p^{2} \operatorname{Trace}\left(\boldsymbol{\Sigma}^{2}\right)}=\frac{1}{p}\left\{1+\frac{\{\operatorname{Trace}(\boldsymbol{\Sigma})\}^{2}}{\operatorname{Trace}\left(\boldsymbol{\Sigma}^{2}\right)}\right\} \\
& =\frac{1}{2 \nu+n+1}\left\{1+\frac{\{\operatorname{Trace}(\overline{\mathbf{G}})\}^{2}}{\operatorname{Trace}\left(\overline{\mathbf{G}}^{2}\right)}\right\}
\end{aligned}
$$

Equation (30) shows that the the normalized standard deviation of $\mathbf{G}$ will be smaller for higher values of $\nu$. For a special case when $\overline{\mathbf{G}}$ is an idempotent matrix or a diagonal matrix (such as the mass matrix of a uniform spring-mass chain), we have

$$
\delta_{G}^{2}=\frac{1}{2 \nu+n+1}\left\{1+\frac{\{n\}^{2}}{n}\right\}=\frac{1+n}{2 \nu+n+1}
$$

This equation clearly shows that for a system with fixed dimension, the uncertainty in the system matrices reduces when $\nu$ increases. Recall that $\nu$ is the order of the inverse moment that we have 
enforced to exist. Intuitively Eq. (30) implies that if we enforce more constraint (in terms of the order of the inverse-moment), the resulting distribution becomes less uncertain. This fact, in turn, allows one to control the amount of uncertainty in the system by choosing different values of $\nu$. It is interesting to observe that the parameter $\nu$, which was originally used as the the order of the inverse-moment constraint, now solely controls the amount of variability in the matrices as both $n$ and $\overline{\mathbf{G}}$ are fixed. If $\delta_{G}^{2}$ is known (e.g., from experiments or experience) then Eq. (30) can be used to calculate $\nu$. Next we consider the properties of the inverse of the system matrices.

\section{Statistical Properties of the Inverse of the System Matrices}

Suppose $\mathbf{F}=\mathbf{G}^{-1}$ denotes the inverse of a system matrix. The jacobian of this transformation ${ }^{20}$ is given by $J(\mathbf{G} \rightarrow \mathbf{F})=|\mathbf{F}|^{-(n+1)}$. Using this, the pdf of the inverse of the system matrices can be obtained from Eq. (22) as

$$
\begin{aligned}
p_{\mathbf{F}}(\mathbf{F})=J(\mathbf{G} & \rightarrow \mathbf{F}) p_{\mathbf{G}}\left(\mathbf{G}=\mathbf{F}^{-1}\right) \\
& =\frac{r^{-n r}}{\Gamma_{n}(r)}|\overline{\mathbf{G}}|^{-r}|\mathbf{F}|^{-(\nu+n+1)} \text { etr }\left\{-r \overline{\mathbf{G}}^{-1} \mathbf{F}^{-1}\right\}, \quad \text { where } \quad r=\nu+\frac{1}{2}(n+1)
\end{aligned}
$$

Comparing this with the inverted Wishart distribution in Eq. (6) we can say that the inverse of a system matrix has a inverted Wishart distribution with parameters $m=2(\nu+n+1)$ and $\mathbf{\Psi}=(2 \nu+n+1) \overline{\mathbf{G}}^{-1}$. From this discussion we have the following basic result:

Theorem 4. If $\nu$-th order inverse-moment of a system matrix $\mathbf{G} \equiv\{\mathbf{M}, \mathbf{C}, \mathbf{K}\}$ exists and only the mean of $\mathbf{G}$ is available, say $\overline{\mathbf{G}}$, then the pdf of $\mathbf{G}^{-1}$ follows the inverted Wishart distribution with parameters $m=2(\nu+n+1)$ and $\mathbf{\Psi}=(2 \nu+n+1) \overline{\mathbf{G}}^{-1}$, that is $\mathbf{G}^{-1} \sim I W_{n}(2(\nu+n+1),(2 \nu+$ $\left.n+1) \overline{\mathbf{G}}^{-1}\right)$.

The exact pdf of the inverse of the system matrices might be useful, for example, to obtain the pdf of the response. At present mostly perturbation based methods are used for this purpose. The first moment (mean), second-moment and the elements of the covariance tensor of $\mathbf{G}^{-1}$ can be obtained ${ }^{18}$ exactly in closed-form as

$$
\begin{aligned}
\mathrm{E}\left[\mathbf{G}^{-1}\right] & =\frac{\mathbf{\Psi}}{m-2 n-2}=\frac{2 \nu+n+1}{2 \nu} \overline{\mathbf{G}}^{-1} \\
\mathrm{E}\left[\mathbf{G}^{-2}\right] & =\frac{\operatorname{Trace}(\boldsymbol{\Psi}) \mathbf{\Psi}+(m-2 n-1) \mathbf{\Psi}^{2}}{(m-2 n-1)(m-2 n-2)(m-2 n-4)} \\
& =\frac{(2 \nu+n+1)^{2} \operatorname{Trace}\left(\overline{\mathbf{G}}^{-1}\right) \overline{\mathbf{G}}^{-1}+2 \nu \overline{\mathbf{G}}^{-2}}{2 \nu(2 \nu+1)(2 \nu-2)} \\
\operatorname{cov}\left(G_{i j}^{-1}, G_{k l}^{-1}\right) & =\frac{2(m-2 n-2)^{-1} \psi_{i j} \psi_{k l}+\psi_{i k} \psi_{j l}+\psi_{i l} \psi_{k j}}{(m-2 n-1)(m-2 n-2)(m-2 n-4)} \\
& =\frac{(2 \nu+n+1)\left(\nu^{-1} \bar{G}_{i j}^{-1} \bar{G}_{k l}^{-1}+\bar{G}_{i k}^{-1} \bar{G}_{j l}^{-1}+\bar{G}^{-1} i l \bar{G}_{k j}^{-1}\right)}{2 \nu(2 \nu+1)(2 \nu-2)}
\end{aligned}
$$

In the above equations $\bar{G}_{i j}^{-1}$ implies $i j$-th element of the $\bar{G}^{-1}$ matrix (that is $\bar{G}_{i j}^{-1} \neq 1 / \bar{G}_{i j}$ ). From Eq. (33) observe that $\nu$ must be more than 0 for the existence of the mean of the inverse matrices. Similarly from Eq. (34), for the existence of the second inverse-moment $\nu$ must be more than 1. This in turn implies that for the distribution given in Theorem 1, neither the mean nor the variance of the inverse of the random system matrix exist. This is however not a limitation because we are 
interested in the existence of the inverse moments of the dynamic stiffness matrix. It is perfectly possible (provided there is some damping in the system) that the inverse-moments of the dynamic stiffness matrix exists while that for the individual system matrices do not.

Equation (33) also shows one intriguing fact. Suppose the degrees-of-freedom of a system $n=100$ and $\nu=2$. Therefore, from Eq. (24) we have $\mathrm{E}[\mathbf{G}]=\overline{\mathbf{G}}$ and from Eq. (33) we have

$$
\mathrm{E}\left[\mathbf{G}^{-1}\right]=\frac{2 \times 2+100+1}{4} \overline{\mathbf{G}}^{-1}=26.25 \overline{\mathbf{G}}^{-1}
$$

This is clearly unacceptable for engineering structural matrices because the randomness of real system are not very large. Of course there is no reason as to why always $\mathrm{E}\left[\mathbf{G}^{-1}\right]=\overline{\mathbf{G}}^{-1}$. However, we do not expect them to be so far apart! One possible way to reduce this 'gap' is to increase the values of $\nu$. However, this implies the reduction of the variance, that is, the assumption of more constraints than necessary. The discrepancy between the mean of the inverse and inverse of the mean of the random matrices appears to be a fundamental limitation of the matrix variate distribution derived so far. A new approach based on optimal Wishart matrices is proposed in the next section to address this issue.

\section{Optimal Wishart Distributions}

From the previous discussions it follows that one could have formulated the maximum entropy approach in terms of the inverse of the systems matrices also. In that case one would obtain a very large difference between the $\mathrm{E}[\mathbf{G}]$ and $\overline{\mathbf{G}}$. It is not quite obvious whether the maximum entropy approach should be formulated with respect $\mathbf{G}$ or $\mathbf{G}^{-1}$ or indeed any other powers of $\mathbf{G}$. For structural vibration problems it is perhaps more logical to formulated the maximum entropy approach in terms of the eigenvalues and eigenvectors of the system. Depending on the what 'information' we select, the resulting distribution can differ dramatically from one to another. To avoid this problem of 'information dependence' in this section an optimal Wishart distribution is proposed.

Here the main idea is that the distribution of $\mathbf{G}$ must be such that $\mathrm{E}[\mathbf{G}]$ and $\mathrm{E}\left[\mathbf{G}^{-1}\right]$ be closest to $\overline{\mathbf{G}}$ and $\overline{\mathbf{G}}^{-1}$ respectively. Suppose $\mathbf{G}$ has Wishart distribution with parameters $p=n+1+\theta$ and $\boldsymbol{\Sigma}=\overline{\mathbf{G}} / \alpha$, that is, $\mathbf{G} \sim W_{n}(n+1+\theta, \overline{\mathbf{G}} / \alpha)$. Recall that the variance of the system is dependent only on $\theta=2 \nu$. Therefore, the aim is to determine the parameter $\alpha \in \mathbb{R}^{+}$such that $\mathrm{E}[\mathbf{G}]$ and $\mathrm{E}\left[\mathbf{G}^{-1}\right]$ become closest to $\overline{\mathbf{G}}$ and $\overline{\mathbf{G}}^{-1}$. To obtain the optimal value of $\alpha$ we define the "normalized errors' as

$$
\begin{aligned}
\varepsilon_{1} & =\|\overline{\mathbf{G}}-\mathrm{E}[\mathbf{G}]\|_{\mathrm{F}} /\|\overline{\mathbf{G}}\|_{\mathrm{F}} \\
\text { and } \quad \varepsilon_{2} & =\left\|\overline{\mathbf{G}}^{-1}-\mathrm{E}\left[\mathbf{G}^{-1}\right]\right\|_{\mathrm{F}} /\left\|\overline{\mathbf{G}}^{-1}\right\|_{\mathrm{F}}
\end{aligned}
$$

Because $\mathbf{G} \sim W_{n}(n+1+\theta, \overline{\mathbf{G}} / \alpha)$ we have

$$
\begin{aligned}
\mathrm{E}[\mathbf{G}] & =\frac{n+1+\theta}{\alpha} \overline{\mathbf{G}} \\
\text { and } \quad \mathrm{E}\left[\mathbf{G}^{-1}\right] & =\frac{\alpha}{\theta} \overline{\mathbf{G}}^{-1}
\end{aligned}
$$

We define the objective function to be minimized as

$$
\chi^{2}=\varepsilon_{1}^{2}+\varepsilon_{2}^{2}
$$

Using Eqs. (37)-(40), this expression can be simplified as

$$
\chi^{2}=\left(1-\frac{n+1+\theta}{\alpha}\right)^{2}+\left(1-\frac{\alpha}{\theta}\right)^{2}
$$

10 of 21 
The optimal value of $\alpha$ can be obtained as by setting

$$
\begin{aligned}
& \quad \frac{\partial \chi^{2}}{\partial \alpha}=0 \\
& \text { or } \quad \alpha^{4}-\alpha^{3} \theta-\theta^{4}+(-2 n+\alpha-2) \theta^{3}+\left((n+1) \alpha-n^{2}-2 n-1\right) \theta^{2}=0
\end{aligned}
$$

This fourth-order equation in $\alpha$ has the following four exact solutions

$$
\alpha= \pm \sqrt{\theta(n+1+\theta)} \text { and } \alpha=\theta / 2 \pm \mathrm{i} \sqrt{\theta(n+1+3 \theta / 4)}
$$

Clearly, the only feasible value of $\alpha \in \mathbb{R}^{+}$is

$$
\alpha=\sqrt{\theta(n+1+\theta)}
$$

The choice of $\alpha$ in Eq. (45) not only minimizes the overall difference, but also the difference between $\mathrm{E}[\mathbf{G}]$ and $\overline{\mathbf{G}}$ and $\mathrm{E}\left[\mathbf{G}^{-1}\right]$ and $\overline{\mathbf{G}}^{-1}$ become the same as

$$
\varepsilon_{1}^{2}=\varepsilon_{2}^{2}=\left(1-\sqrt{\frac{n+1+\theta}{\theta}}\right)^{2}
$$

This discussion implies that if $\mathbf{G} \sim W_{n}(n+1+\theta, \overline{\mathbf{G}} / \sqrt{\theta(n+1+\theta)})$, then the resulting distribution becomes independent of whether $\mathbf{G}$ or $\mathbf{G}^{-1}$ is used for the derivation of the distribution. As a result we have the following:

Theorem 5. If $\nu$-th order inverse-moment of a system matrix $\mathbf{G} \equiv\{\mathbf{M}, \mathbf{C}, \mathbf{K}\}$ exists and only the mean of $\mathbf{G}$ is available, say $\overline{\mathbf{G}}$, then the unbiased distribution of $\mathbf{G}$ follows the Wishart distribution with parameters $p=(2 \nu+n+1)$ and $\boldsymbol{\Sigma}=\overline{\mathbf{G}} / \sqrt{2 \nu(2 \nu+n+1)}$, that is

$\mathbf{G} \sim W_{n}(2 \nu+n+1, \overline{\mathbf{G}} / \sqrt{2 \nu(2 \nu+n+1)})$.

To give a numerical illustration, again consider $n=100$ and $\nu=2$, so that $\theta=2 \nu=4$. For the distribution in the previous section $\alpha=2 \nu+n+1=105$. For the optimal distribution, $\alpha=\sqrt{\theta(\theta+n+1)}=2 \sqrt{105}=20.49$. Using these values we have $\mathrm{E}[\mathbf{G}]=\frac{105}{2 \sqrt{105}} \overline{\mathbf{G}}=5.12 \overline{\mathbf{G}}$ and $\mathrm{E}\left[\mathbf{G}^{-1}\right]=\frac{2 \sqrt{105}}{4} \overline{\mathbf{G}}^{-1}=5.12 \overline{\mathbf{G}}^{-1}$. The overall normalized difference for the previous case is $\chi^{2}=$ $0+(1-105 / 4)^{2}=637.56$. The same for the optimal distribution is $\chi^{2}=2(1-\sqrt{105} / 2)^{2}=34.01$, which is considerable smaller compared to the non-optimal distribution.

\section{Numerical Examples}

\section{A. Example 1: A Two Degree-of-Freedom System}

We consider a simple two-degrees-of-freedom system to illustrate the matrix variate distribution derived in section III. The system considered is shown in figure 1 with the mean values of the system parameters. The mean of the mass, damping and stiffness matrices are given by

$$
\overline{\mathbf{M}}=\left[\begin{array}{cc}
m_{1} & 0 \\
0 & m_{2}
\end{array}\right], \quad \overline{\mathbf{C}}=\left[\begin{array}{cc}
c_{1}+c_{2} & -c_{2} \\
-c_{2} & c_{2}
\end{array}\right], \quad \text { and } \quad \overline{\mathbf{K}}=\left[\begin{array}{cc}
k_{1}+k_{2} & -k_{2} \\
-k_{2} & k_{2}+k_{3}
\end{array}\right]
$$

For this system $n=2$ and we assume that the second-order inverse moments of the system matrices exist so that $\nu=2$. Using Theorem 2 it follows that $\mathbf{G} \sim W_{2}(7, \overline{\mathbf{G}} / 7)$. Suppose $\mathbf{Z} \in \mathbb{R}_{2}^{+}$is a symmetric positive definite matrix such that

$$
\mathbf{Z}=\left[\begin{array}{ll}
z_{11} & z_{12} \\
z_{12} & z_{22}
\end{array}\right]
$$

11 of 21 


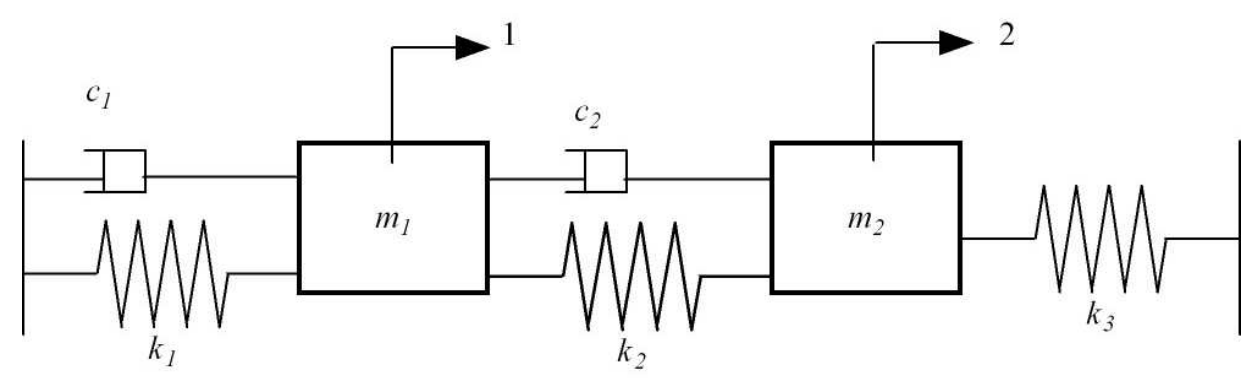

Figure 1. The mean model of a two-degree-of-freedom damped system.

The positive definite condition requires that $|\mathbf{Z}|>0$, that is the variables $z_{11}, z_{12}$ and $z_{22}$ should vary such that $z_{11} z_{22}-z_{12}^{2}>0$. Note that $\mathbf{Z}$ becomes a diagonal matrix for the distribution of the mass matrix. Using the expression of the pdf of the Wishart matrix in Eq. (4) we have

$$
\begin{aligned}
p_{\mathbf{G}}(\mathbf{Z}) & =\left\{2^{7} \Gamma_{2}\left(\frac{1}{2} 7\right)|\overline{\mathbf{G}} / 7|^{\frac{1}{2} 7}\right\}^{-1}|\mathbf{Z}|^{\frac{1}{2}(4)} \operatorname{etr}\left\{-\frac{1}{2}(\overline{\mathbf{G}} / 7)^{-1} \mathbf{Z}\right\} \\
& =(7 / 2)^{7} \frac{4}{15 \pi}|\overline{\mathbf{G}}|^{-7 / 2}\left(z_{11} z_{22}-z_{12}^{2}\right)^{2} \operatorname{etr}\left\{-\frac{7}{2} \overline{\mathbf{G}}^{-1} \mathbf{Z}\right\}
\end{aligned}
$$

Using Eq. (49), the pdf of the mass matrix can be obtained as

$$
\begin{aligned}
p_{\mathbf{M}}(\mathbf{Z}) & =\frac{823543}{480 \pi}|\overline{\mathbf{M}}|^{-7 / 2}\left(z_{11} z_{22}\right)^{2} \operatorname{etr}\left\{-\frac{7}{2} \overline{\mathbf{M}}^{-1} \mathbf{Z}\right\} \\
& =\frac{823543}{480 \pi} \frac{\left(z_{11} z_{22}\right)^{2}}{\left(m_{1} m_{2}\right)^{3} \sqrt{m_{1} m_{2}}} \exp \left\{-\frac{7}{2} \frac{z_{11} m_{2}+z_{22} m_{1}}{m_{1} m_{2}}\right\} ; \quad z_{11} z_{22}>0
\end{aligned}
$$

Equation (50) is the joint pdf of the two diagonal elements of the mass matrix. Similarly, the pdfs of the damping and stiffness matrices can be expressed as

$$
p_{\mathbf{C}}(\mathbf{Z})=\frac{823543}{480 \pi} \frac{\left(z_{11} z_{22}-z_{12}^{2}\right)^{2}}{\left(c_{1} c_{2}\right)^{3} \sqrt{c_{1} c_{2}}} \exp \left\{-\frac{7}{2} \frac{z_{11} c_{2}+2 z_{12} c_{2}+z_{22}\left(c_{1}+c_{2}\right)}{c_{2} c_{1}}\right\} ; \quad z_{11} z_{22}-z_{12}^{2}>0
$$

and

$$
\begin{aligned}
& p_{\mathbf{K}}(\mathbf{Z})=\frac{823543}{480 \pi} \frac{\left(z_{11} z_{22}-z_{12}^{2}\right)^{2}}{\left(k_{1} k_{2}+k_{1} k_{3}+k_{2} k_{3}\right)^{7 / 2}} \times \\
& \exp \left\{-\frac{7}{2} \frac{z_{11}\left(k_{2}+k_{3}\right)+2 z_{12} k_{2}+z_{22}\left(k_{1}+k_{2}\right)}{k_{1} k_{2}+k_{1} k_{3}+k_{2} k_{3}}\right\} ; \quad z_{11} z_{22}-z_{12}^{2}>0
\end{aligned}
$$

This simple example is for illustrative purpose only. The non-parametric uncertainty modeling method proposed here is ideally suitable for large complex systems undergoing mid and high frequency vibration. Theorem 3 allows a simple method to generate the samples of the system matrices. From these samples it is possible to propagate the uncertainty in complex systems using Monte Carlo simulation as shown in the next example.

\section{B. Example 2: Dynamic Response of a Clamped Plate With Random Properties}

A cantilever plate with a slot is considered in this section. The diagram of the plate together with the deterministic numerical values assumed for the system parameters are shown in figure 2 . The

12 of 21

American Institute of Aeronautics and Astronautics 


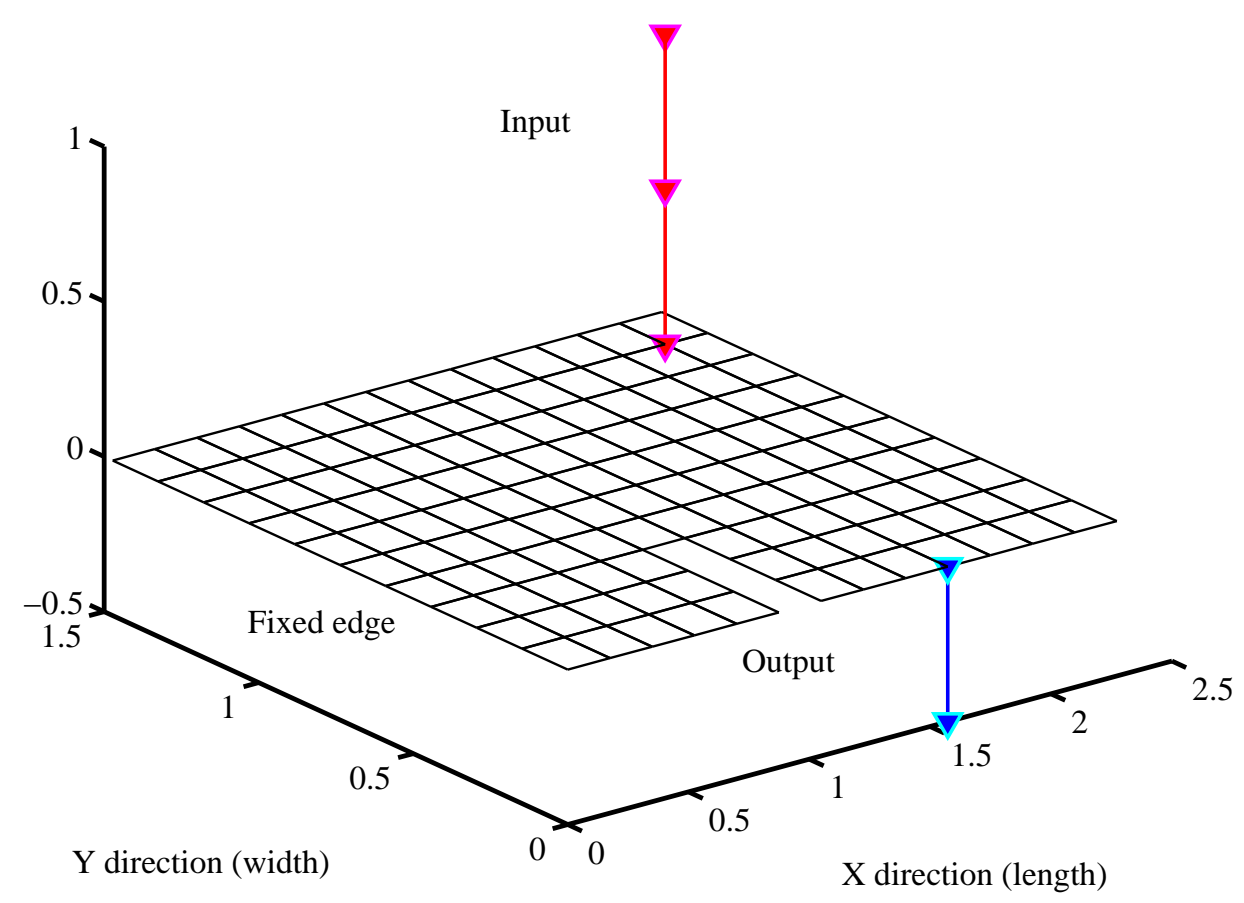

Figure 2. A Cantilever plate with a slot: $\mu=0.3, \rho=8000 \mathrm{~kg} / \mathrm{m}^{3}, t=5 \mathrm{~mm}, L_{x}=2.27 \mathbf{m}, L_{y}=1.47 \mathbf{m}$.

plate is excited by an unit harmonic force and the response is calculated at the points shown in the diagram. The standard four-noded thin plate bending element (resulting 12 dof per element) is used. The plate is divided into 13 elements in x-axis and 10 elements in the y-axis for the numerical calculations. The resulting system has 429 degrees-of-freedom so that $n=429$. The first six deterministic mode shapes and natural frequencies of the plate are shown in figure 3 . The amplitude of the cross frequency response function (FRF) and the driving point FRF of the deterministic plate is shown in figure 4. It is assumed that the modal damping factors are uniformly distributed between $1 \%$ to $4 \%$ (with a mean of $2.5 \%$ ).

Now we consider uncertainties in the plate structure. It is assumed that the plate is 'loaded' at random points with 30 masses, each weighting $0.5 \%$ of the total mass of the plate. Therefore, the total amount of the mass distributed over 30 randomly selected nodes is $0.15 \%$ of the total mass of the plate. A 500-sample Monte Carlo simulation is performed to obtain the FRFs. Figure 5 shows the direct finite-element (FE) Monte Carlo simulation result for the cross-FRF. Realizations of the amplitude of the FRF for each sample are shown together with the ensemble mean, $5 \%$ and $95 \%$ probability points. The ensemble mean follows the deterministic result very closely across the range of the excitation frequency considered in this study. Figure 6 shows the direct finite-element Monte Carlo simulation result for the driving-point-FRF. Realizations of the amplitude of the FRF for each sample are shown together with the ensemble mean, $5 \%$ and $95 \%$ probability points. The amount of 'variation' in this case is smaller compared to the cross-FRF. This is due to the fact that randomly placed masses only have a significant effect on the driving point FRF if the masses are close to the forcing point. While for the cross-FRF, all the masses located between (and around) the two points can affect the FRF. Because the probability of occurrence of the later event is more than the first one, we see a greater variability in the cross-FRF compared to the driving-point FRF.

Now we want to see if the results produced by the direct finite element MCS can be reproduced

13 of 21

American Institute of Aeronautics and Astronautics 

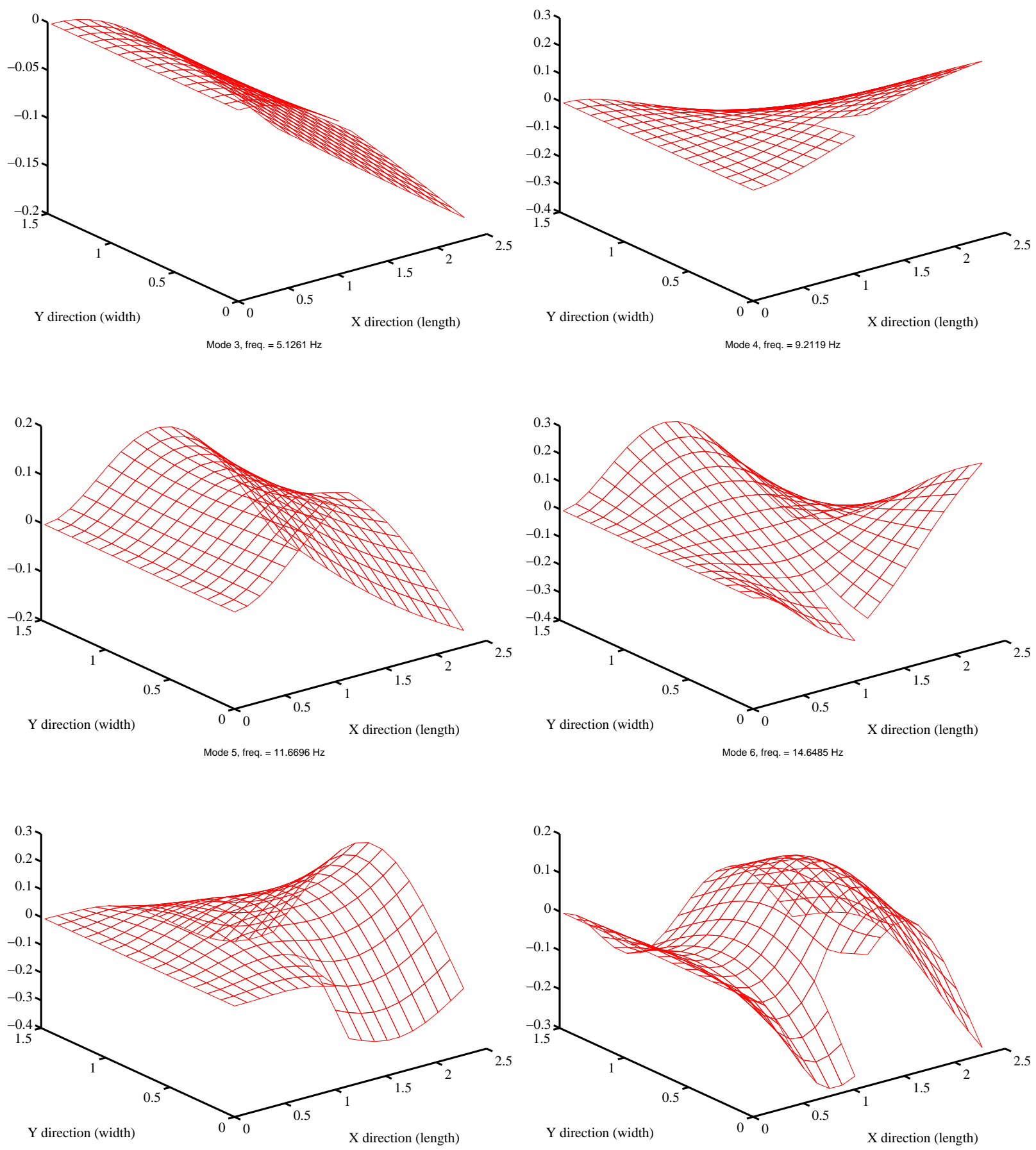

Figure 3. First six deterministic mode shapes and natural frequencies of the plate.

using the random matrix theory. The details of the parametric variations of the locations of the randomly placed masses will not be used in the random matrix approach. This example is aimed to depict a realistic situation when the detailed information regarding the uncertainty of a complex engineering system may not be available to the analyst. From the simulated random mass matrices we obtain $\delta_{M}=2.0449$, which is the only uncertainty related information to be used in

\section{4 of 21}




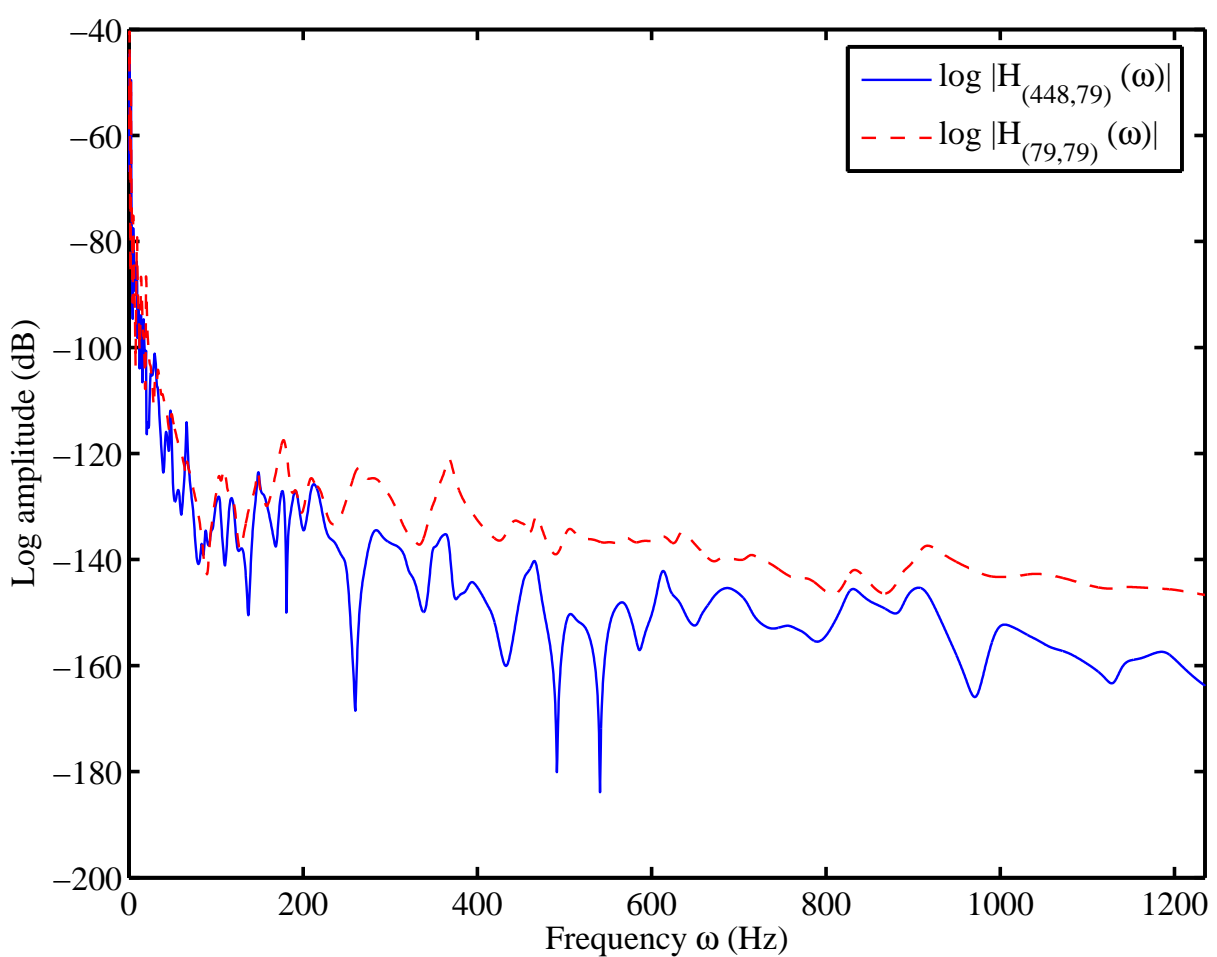

Figure 4. The amplitude of the driving-point FRF and cross FRF of the deterministic plate (node numbers correspond to the numbers before applying the boundary conditions).

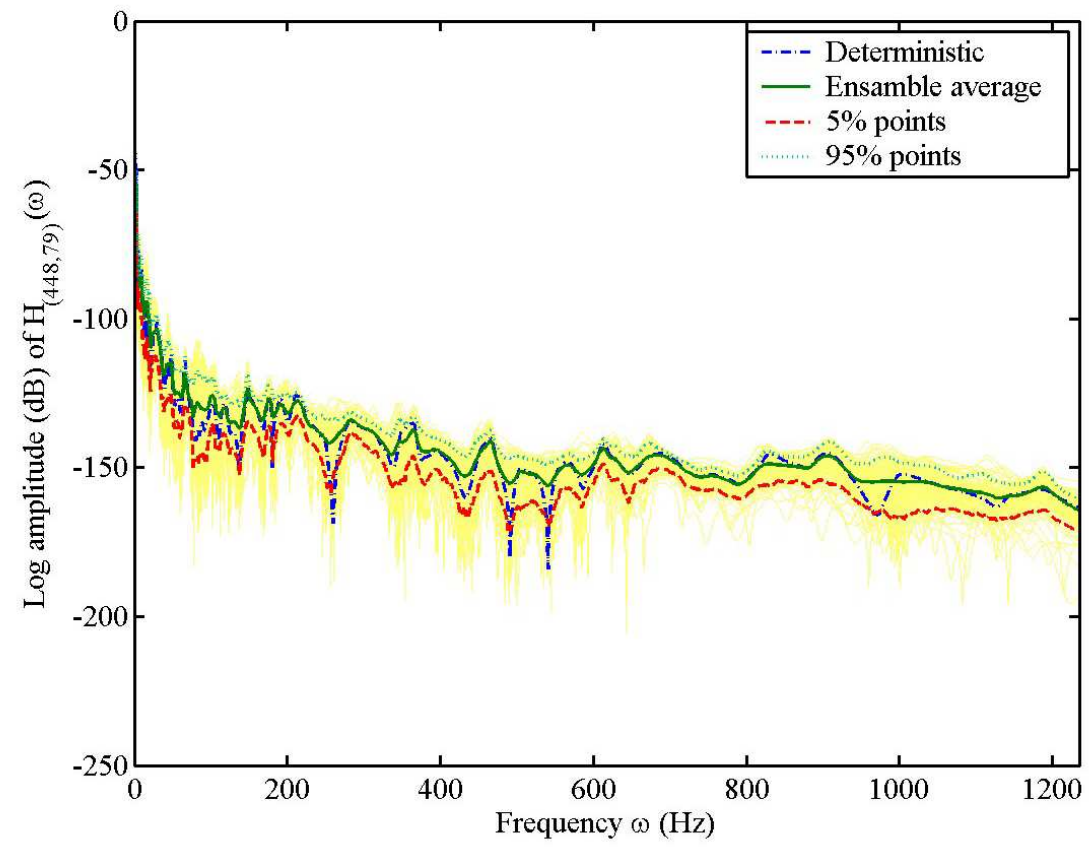

Figure 5. Direct finite-element MCS of the amplitude of the cross-FRF of the plate with randomly placed masses; 30 masses, each weighting $0.5 \%$ of the total mass of the plate are simulated.

15 of 21

American Institute of Aeronautics and Astronautics 


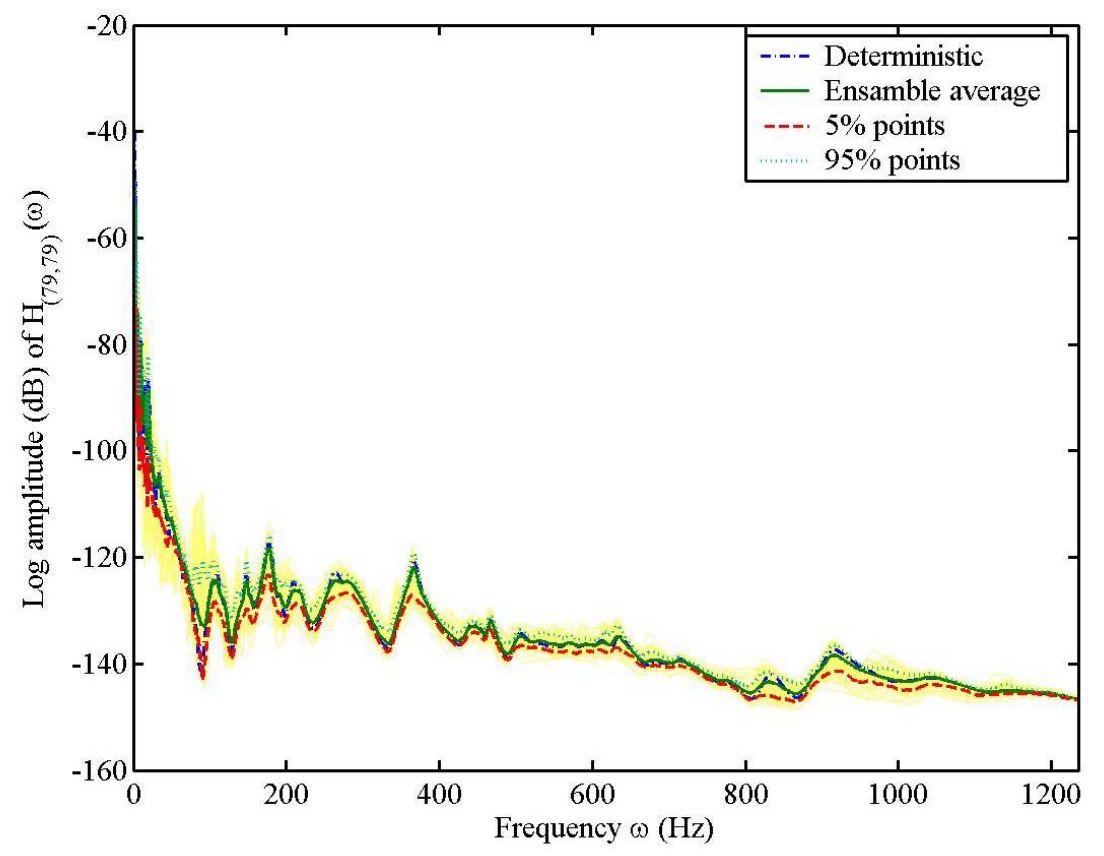

Figure 6. Direct finite-element MCS of the amplitude of the driving-point FRF of the plate with randomly placed masses; 30 masses, each weighting $0.5 \%$ of the total mass of the plate are simulated.

the random matrix approach. Here $\delta_{K}=0$ and $\delta_{C}=0$ and $n=429$. Using this data the samples of the mass matrices are simulated using the optimal Wishart matrices derived in section IV. The simulation procedure is based on the simulation of the Gaussian random matrices as described in Theorem 3. Alternatively MATLAB ${ }^{\circledR}$ command wishrnd can be used to generate the samples of Wishart matrices.

Figure 7 shows the Monte Carlo simulation result for the cross-FRF using the optimal Wishart mass matrix. Realizations of the amplitude of the FRF of each sample are shown in the figure along with the ensemble mean, $5 \%$ and $95 \%$ probability points. Figure 8 shows the Monte Carlo simulation result for the driving-point-FRF using the optimal Wishart mass matrix. Realizations of the amplitude of the FRF of each sample are shown in the figure along with the ensemble mean, $5 \%$ and $95 \%$ probability points. The amount of 'variation' in this case is smaller compared to the cross-FRF, but not as small as the direct FE simulation results.

The predicted mean values using the direct FE simulation and the random matrix theory (RMT) are compared in figure 9 and figure 10 for the cross-FRF and driving-point-FRF respectively. The results obtained from the random matrix theory (RMT) goes through the results obtained using FE simulation. The predicted $5 \%$ and $95 \%$ probability points using the direct FE simulation and random matrix theory (RMT) are compared in figure 11 and figure 12. The results obtained for the cross-FRF agree better than the driving-point-FRF. Considering the fact that the only uncertainty related information used in the random matrix method is $\delta_{M}$, these results are encouraging.

\section{Summary and Conclusions}

A non-parametric method for uncertainty quantification in linear dynamic systems has been proposed. The method is based on the maximum entropy principle and random matrix theory. It is assumed that only the mean of the system matrices are known. The derived probability density

16 of 21

American Institute of Aeronautics and Astronautics 


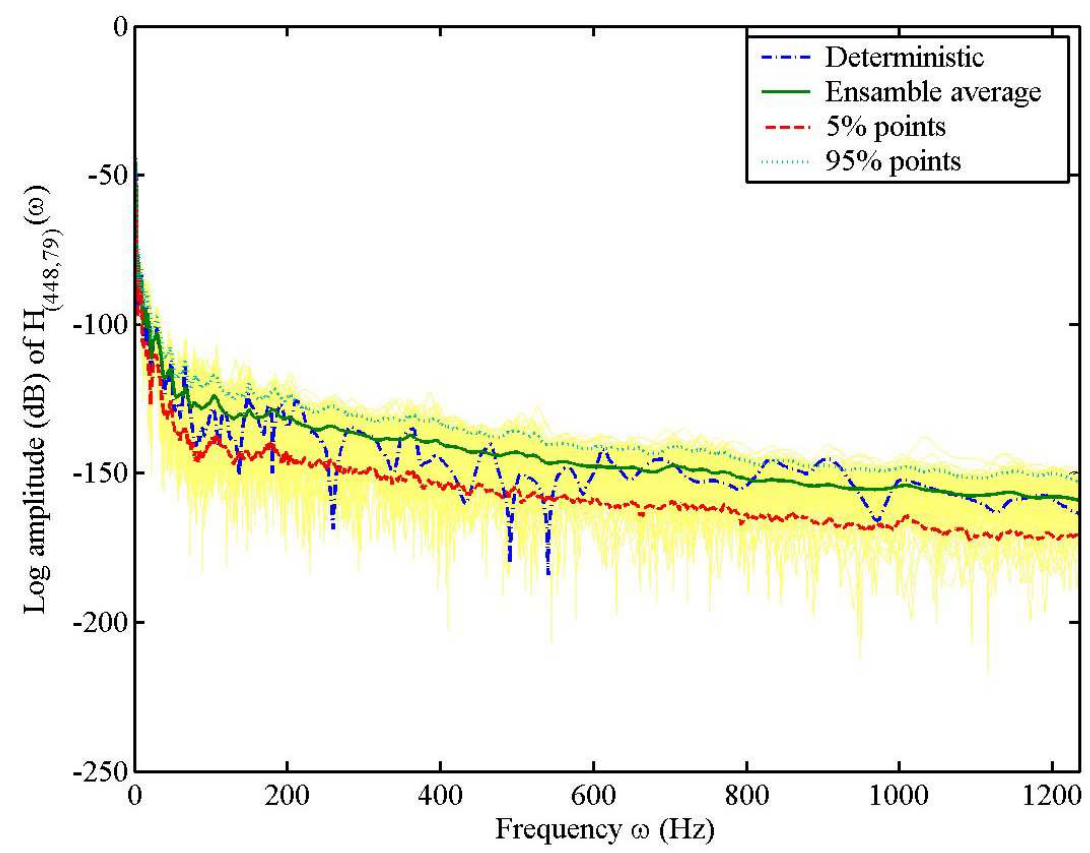

Figure 7. MCS of the amplitude of the cross-FRF of the plate using optimal Wishart mass matrix, $n=429, \delta_{M}=2.0449$.

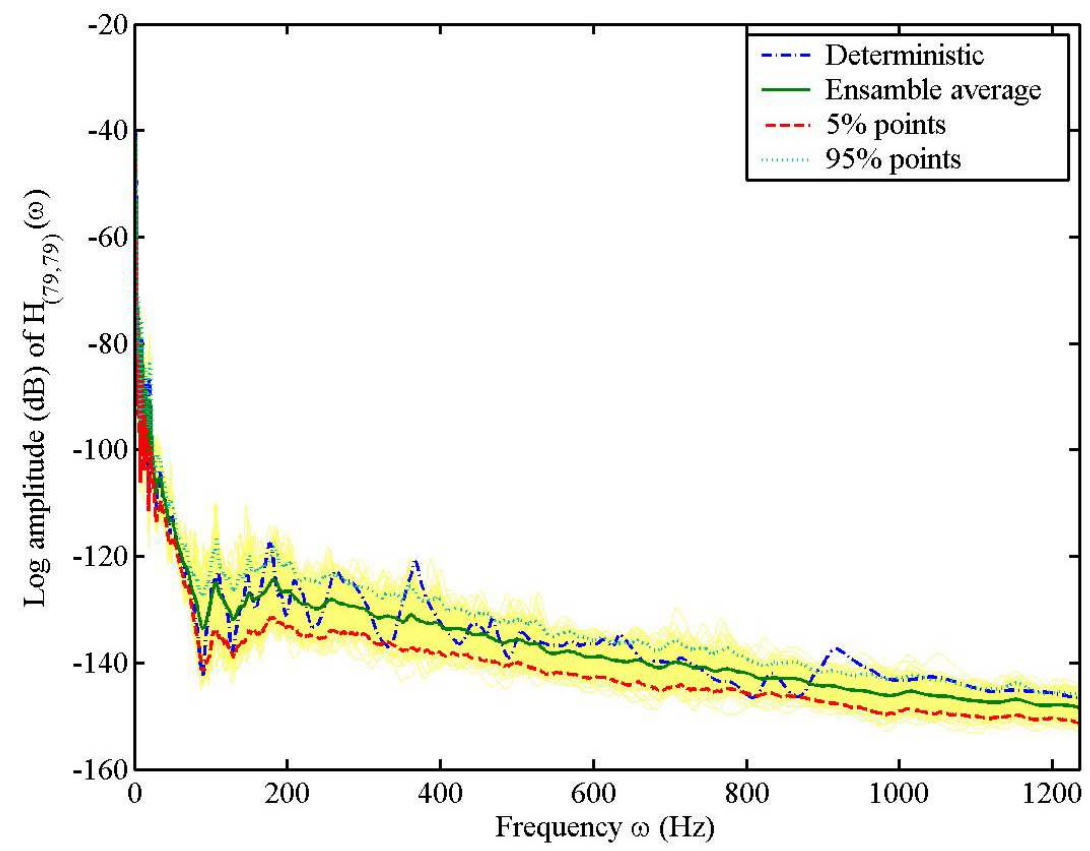

Figure 8. MCS of the amplitude of the driving-point-FRF of the plate using optimal Wishart mass matrix, $n=429, \delta_{M}=2.0449$.

function of the random system matrices are characterized by the dimension of the matrices, their mean values and a scalar parameter defining their randomness. The main outcome of this study is

\section{7 of 21}

American Institute of Aeronautics and Astronautics 


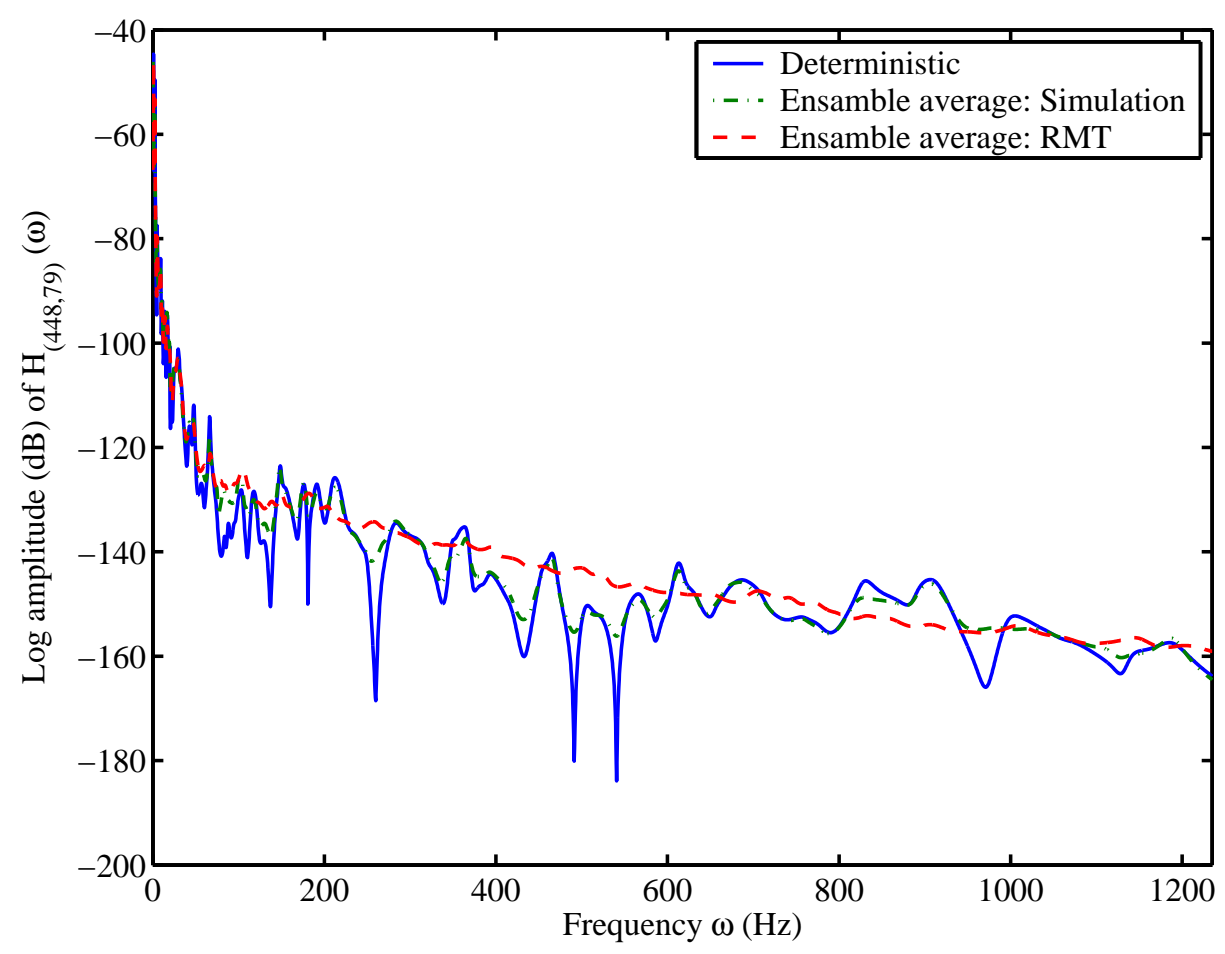

Figure 9. Comparison of the mean values of the amplitude of the cross-FRF.

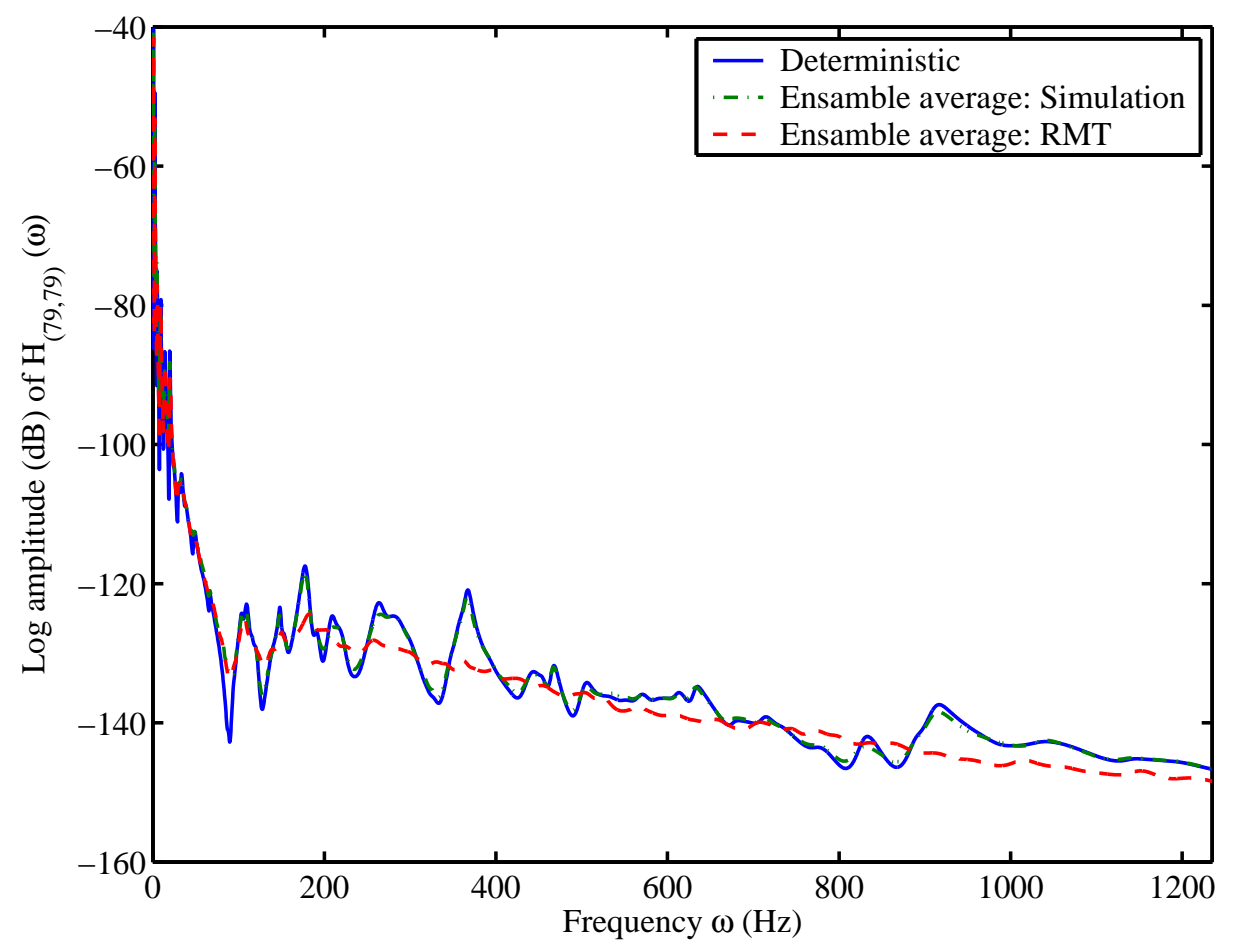

Figure 10. Comparison of the mean values of the amplitude of the driving-point-FRF.

that if only the mean value of a system matrix is known then the matrix follows a Wishart distribution (with proper parameters). The optimal parameters of the Wishart matrices are obtained such

18 of 21

American Institute of Aeronautics and Astronautics 


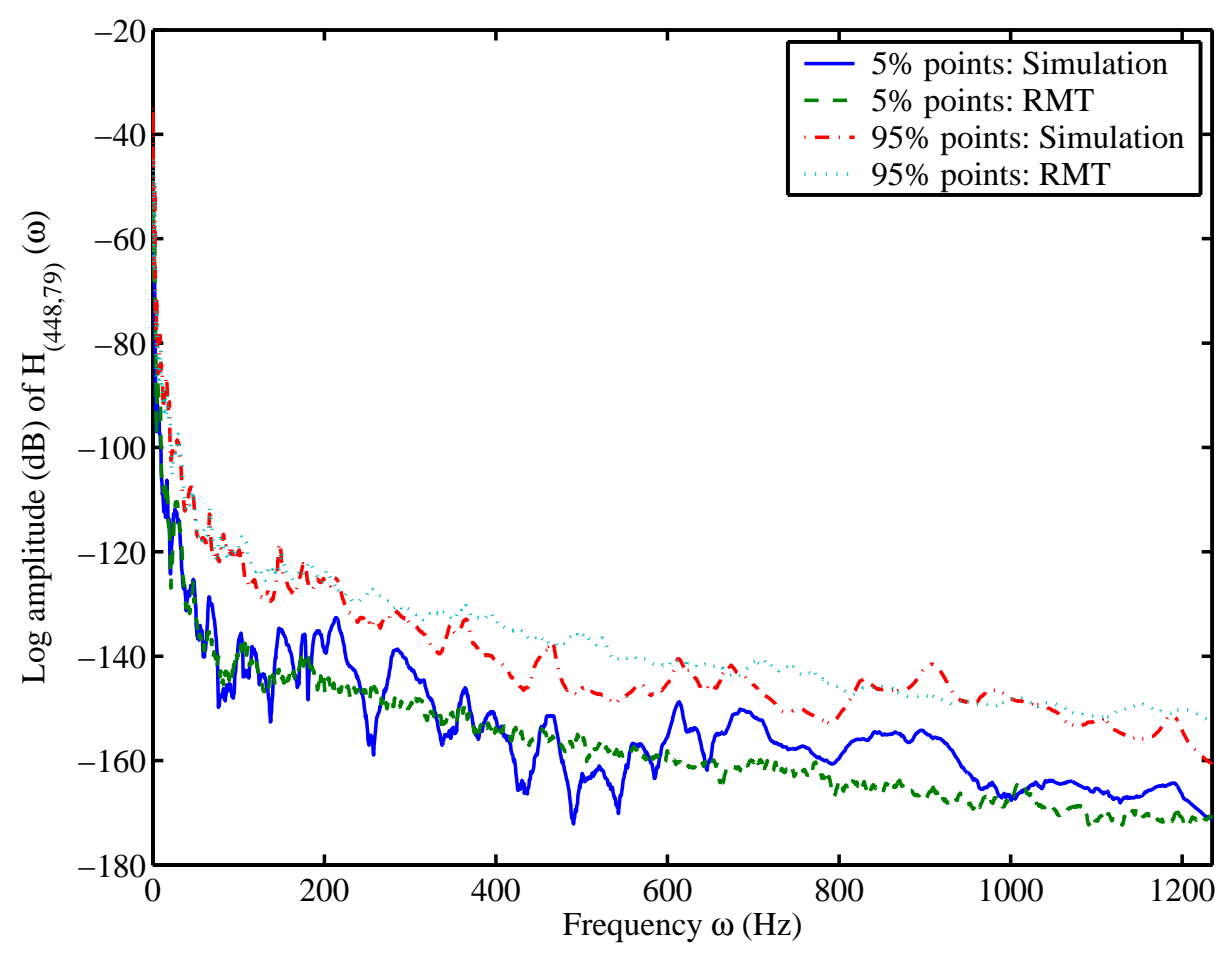

Figure 11. Comparison of the $5 \%$ and $95 \%$ probability points of the amplitude of the cross-FRF.

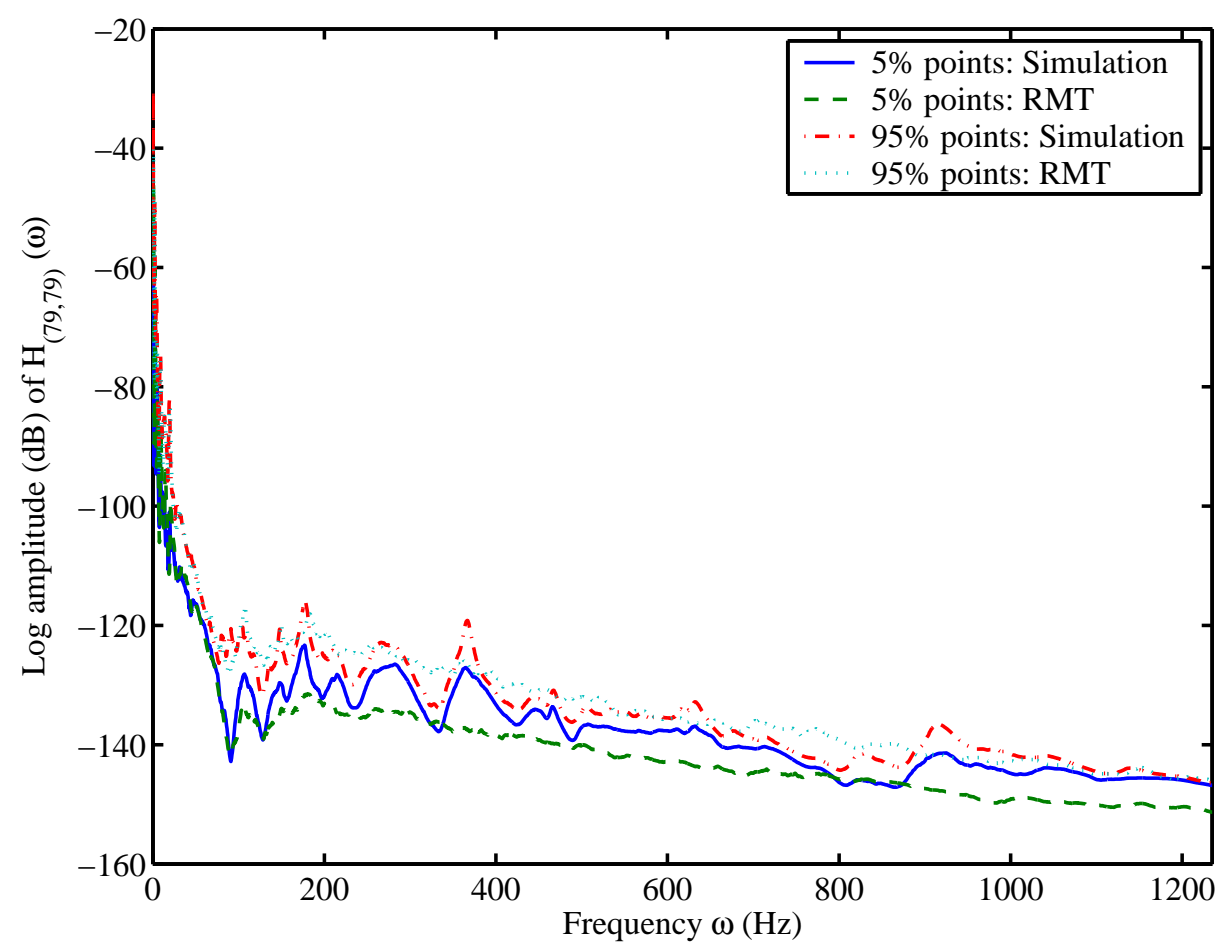

Figure 12. Comparison of the $5 \%$ and $95 \%$ probability points of the amplitude of the driving-pointFRF.

that the mean of the matrix and its inverse produce minimum deviations from their deterministic

19 of 21

American Institute of Aeronautics and Astronautics 
values.

The discovery of the Wishart distribution in the context of structural dynamics is important. This is not only because the Wishart matrices are fundamental to the random matrix theory, but also due to the fact that analysis became simpler with this distribution. The moments and elements of the covariance tensor of the matrix elements and its inverse are obtained exactly in closed-form. The exact expression of the probability density function of the inverse of a random system matrix is derived in closed-from. The applications of the derived matrix variate distribution are illustrated by a two-degree-of-freedom system and a random plate problem with 429 degrees-of-freedom. It is shown that it is possible to predict the variation of the dynamic response using the optimal Wishart distribution. The random matrix based uncertainty quantification and propagation tool developed here do not require explicit information regarding the detailed nature of the uncertainty in the system.

\section{Acknowledgments}

The author acknowledges the support of the UK Engineering and Physical Sciences Research Council (EPSRC) through the award of an Advanced Research Fellowship, grant number GR/T03369/01.

\section{References}

${ }^{1}$ Graham, A., Kronecker Products and Matrix Calculus With Applications, Mathematics and its Applications, Ellis Horwood Limited, Chichester, UK, 1981.

${ }^{2}$ Ghanem, R. and Spanos, P., Stochastic Finite Elements: A Spectral Approach, Springer-Verlag, New York, USA, 1991.

${ }^{3}$ Kleiber, M. and Hien, T. D., The Stochastic Finite Element Method, John Wiley, Chichester, 1992.

${ }^{4}$ Elishakoff, I. and Ren, Y. J., Large Variation Finite Element Method for Stochastic Problems, Oxford University Press, Oxford, U.K., 2003.

${ }^{5}$ Lyon, R. H. and Dejong, R. G., Theory and Application of Statistical Energy Analysis, Butterworth-Heinmann, Boston, 2nd ed., 1995.

${ }^{6}$ Langley, R. S., "A general derivation of the statistical energy analysis equations for coupled dynamic-systems," Journal of Sound and Vibration, Vol. 135, No. 3, December 1989, pp. 499-508.

${ }^{7}$ Langley, R. S. and Bremner, P., "A hybrid method for the vibration analysis of complex structural-acoustic systems," Journal of the Acoustical Society of America, Vol. 105, No. 3, March 1999, pp. 1657-1671.

${ }^{8}$ Sarkar, A. and Ghanem, R., "Mid-frequency structural dynamics with parameter uncertainty," Computer Methods in Applied Mechanics and Engineering, Vol. 191, No. 47-48, 2002, pp. 5499-5513.

${ }^{9}$ Wishart, J., "The generalized product moment distribution in samples from a normal multivariate population," Biometrika, Vol. 20, No. A, 1928, pp. 32-52.

${ }^{10}$ Wigner, E., "On the distribution of roots of certain symmetric matrices," Annals of Mathematics, Vol. 67, 1958, pp. 323-327.

${ }^{11}$ Mezzadri, F. and Snaith, N. C., editors, Recent Perspectives in Random Matrix Theory and Number Theory, London Mathematical Society Lecture Note, Cambridge, U. K., 2005, Cambridge University Press.

${ }^{12}$ Tulino, A. M. and Verdú, S., Random Matrix Theory and Wireless Communications, now Publishers Inc., Hanover, MA, USA, 2004.

${ }^{13}$ Muirhead, R. J., Aspects of Multivariate Statistical Theory, John Wiely and Sons, New York, USA, 1982.

${ }^{14}$ Mehta, M. L., Random Matrices, Academic Press, San Diego, CA, 2nd ed., 1991.

${ }^{15}$ Soize, C., "A nonparametric model of random uncertainties for reduced matrix models in structural dynamics," Probabilistic Engineering Mechanics, Vol. 15, 2000, pp. 277-294.

${ }^{16}$ Soize, C., "Maximum entropy approach for modeling random uncertainties in transient elastodynamics," Journal of the Acoustical Society of America, Vol. 109, No. 5, May 2001, pp. 1979-1996.

${ }^{17}$ Soize, C., "Random matrix theory for modeling uncertainties in computational mechanics," Computer Methods in Applied Mechanics and Engineering, Vol. 194, 2005, pp. 1333-1366.

${ }^{18}$ Gupta, A. and Nagar, D., Matrix Variate Distributions, Monographs \& Surveys in Pure \& Applied Mathematics, Chapman \& Hall/CRC, London, 2000.

${ }^{19}$ Kapur, J. N. and Kesavan, H. K., Entropy Optimization Principles With Applications, Academic Press, San Diego, CA, 1992.

20 of 21 
${ }^{20}$ Mathai, A. M., Jacobians of Matrix Transformation and Functions of Matrix Arguments, World Scientific, London, 1997.

${ }^{21}$ Steeb, W.-H., Matrix Calculus and the Kronecker Product with Applications and C++ Programs, World Scientific Publishing, London, 1997.

${ }^{22}$ Mathai, A. M. and Provost, S. B., Quadratic Forms in Random Variables: Theory and Applications, Marcel Dekker, Inc., 270 Madison Avenue, New York, NY 10016, USA, 1992. 